\title{
Thermal origin of quasilocalized excitations in glasses
}

\author{
Wencheng Ji $\odot$, Tom W. J. de Geus $\odot$, Marko Popović, Elisabeth Agoritsas $₫$, and Matthieu Wyart \\ Institute of Physics, École Polytechnique Fédérale de Lausanne (EPFL), CH-1015 Lausanne, Switzerland
}

(Received 13 July 2020; accepted 11 November 2020; published 4 December 2020)

\begin{abstract}
Key aspects of glasses are controlled by the presence of excitations in which a group of particles can rearrange. Surprisingly, recent observations indicate that their density is dramatically reduced and their size decreases as the temperature of the supercooled liquid is lowered. Some theories predict these excitations to cause a gap in the spectrum of quasilocalized modes of the Hessian that grows upon cooling, while others predict a pseudogap $D_{L}(\omega) \sim \omega^{\alpha}$. To unify these views and observations, we generate glassy configurations of controlled gap magnitude $\omega_{c}$ at temperature $T=0$, using so-called breathing particles, and study how such gapped states respond to thermal fluctuations. We find that (i) the gap always fills up at finite $T$ with $D_{L}(\omega) \approx A_{4}(T) \omega^{4}$ and $A_{4} \sim \exp \left(-E_{a} / T\right)$ at low $T$, (ii) $E_{a}$ rapidly grows with $\omega_{c}$, in reasonable agreement with a simple scaling prediction $E_{a} \sim \omega_{c}^{4}$ and (iii) at larger $\omega_{c}$ excitations involve fewer particles, as we rationalize, and eventually become stringlike. We propose an interpretation of mean-field theories of the glass transition, in which the modes beyond the gap act as an excitation reservoir, from which a pseudogap distribution is populated with its magnitude rapidly decreasing at lower $T$. We discuss how this picture unifies the rarefaction as well as the decreasing size of excitations upon cooling, together with a stringlike relaxation occurring near the glass transition.
\end{abstract}

DOI: 10.1103/PhysRevE.102.062110

\section{INTRODUCTION}

A key feature of structural glasses is that groups of particles can rearrange locally between two metastable states. This motion can be triggered by quantum or thermal fluctuations, or mechanically by exerting an external stress or strain. Such rearrangements are associated with different excitations. At low temperature the dominant source of excitations are two-level systems (TLS) that stem from quantum tunneling between the metastable states [1-3]. At higher temperatures, relaxation in supercooled liquids near the glass transition occurs via thermally activated events, observed to become more and more stringlike upon cooling [4,5]. Upon mechanical loading, at any temperature below the glass transition, plasticity occurs when a group of particles becomes unstable. In the potential energy landscape, this corresponds to a saddle-node bifurcation [6,7] and leads to a rearrangement denoted shear transformation [8]. Understanding how temperature or system preparation controls the density of these excitations remains a challenge. It is, however, a question of practical importance since: (i) the density of shear transformations controls for instance the glass brittleness [9-11], (ii) the rarefaction of activated events near the glass transition controls its fragility [12], and (iii) the density of TLS (recently observed to be almost absent in ultrastable glasses $[13,14]$ ) effects the decoherence in qubits [15] important for quantum computing. Finally, the possible unification of these excitations into a common description is a fundamental problem for a prospective theory of glasses.

These localized excitations should affect the low-frequency spectrum of the Hessian of the energy landscape, since groups of particles that can easily rearrange tend to have a small linear restoring force $[6,16,17]$. Indeed, in numerical glasses, such quasilocalized modes are found at low frequencies [18]. Recently there has been a considerable effort to analyze them [19-25]. In most glasses, it is found that in inherent structures [26], the density of quasilocalized modes $D_{L}(\omega) \approx A_{4}(T) \omega^{4}$, with $\omega$ the frequency. Most remarkably, $A_{4}(T)$ is reduced by several decades as $T$ is reduced by $30 \%$ [25,27] (a similar finding was obtained for the density of TLS [28]). Furthermore, quasilocalized modes also display a lower participation ratio at lower $T$. A unifying explanation for these facts is currently missing [36]. On the theoretical side, two distinct approaches have been proposed. On the one hand, the $\omega^{4}$ power law has been rationalized by making specific assumptions on the disorder and by assuming modes as noninteracting [29,30], or by modeling a quench from $T=\infty$ and including interactions [17]. On the other hand, in mean-field calculations in infinite dimensions for temperatures below the mode-coupling temperature $T_{c}[31]$ the spectrum of the Hessian becomes gapped (excluding obvious long-wave-length Goldstone modes that are always present). Below $T_{c}$, the gap is predicted to grow as $T$ decreases [32-34]. A gap was also predicted from real-space stability arguments in finite dimensions for continuously polydisperse particles, at very low energies and zero temperature [35]. Nevertheless, it is currently unclear if a gap truly exists in finite dimension and at finite temperature.

In this article we seek a unifying scenario for these facts and different approaches, by studying the stability of gapped spectra with respect to thermal fluctuations. Specifically, we use breathing particles $[36,37]$ in order to generate athermal 


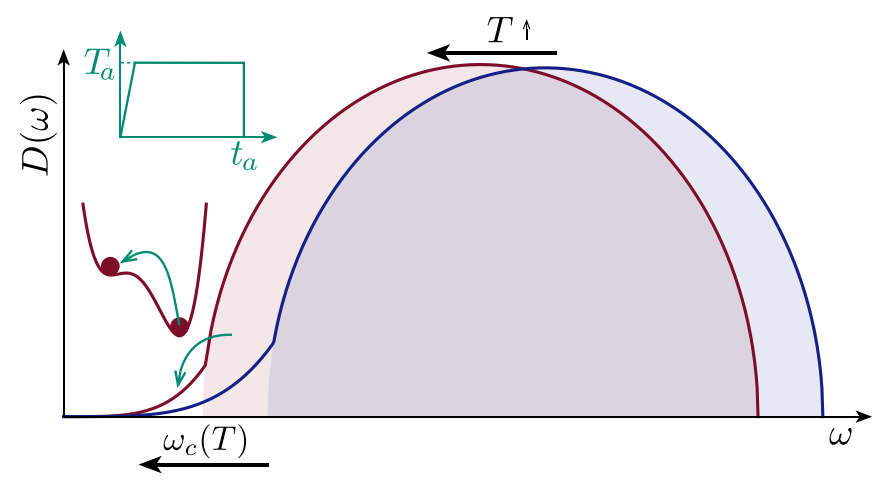

FIG. 1. Schematic density of states for an equilibrated liquid at temperature $T$. When a gapped glass is heated to a temperature $T_{a}$ for a duration $t_{a}$, as sketched in the inset, modes beyond the gap act as a reservoir of excitations that can be thermally activated. It fills up the gap, leading, for small $\omega$, to a pseudogap $D(\omega) \approx D_{L}(\omega) \approx A_{4} \omega^{4}$. This effect is exponentially diminished if $\omega_{c}$ increases (corresponding to a decrease of $T$ as predicted by the infinite-dimensional mean-field description near the glass transition).

ultrastable glasses of controlled gap magnitude $\omega_{c}$. Then, as sketched in Fig. 1, we transiently reheat these glasses, with a standard molecular dynamics simulation, at a low temperature $T_{a}$ for a duration $t_{a}$, before quenching them back to zero temperature. Our central results are that (a) thermal fluctuations, even small, destroy the gap and we recover a density $D_{L}(\omega) \approx A_{4} \omega^{4}$; the prefactor $A_{4}\left(T_{a}, t_{a}\right)$ depends very mildly on $t_{a}$ but presents an Arrhenius dependence on temperature with $A_{4} \sim \exp \left(-E_{a} / T_{a}\right.$ ) (in our temperature units the Boltzmann constant $k_{B}=1$ ). (b) The activation energy $E_{a}$ rapidly increases with the gap magnitude $\omega_{c}$. (c) We introduce an algorithm to decompose the rearrangements into elementary excitations, and find that they involve fewer particles for larger gap values, and eventually become stringlike for our largest gap. We propose a scaling argument for their decreasing size. Overall, these results suggest to describe equilibrated liquids perturbatively as gapped states decorated by thermally activated excitations whose characteristic energy is controlled by the gap itself, leading to a contribution with $A_{4} \sim \exp \left(-E_{a}\left(\omega_{c}(T)\right) / T\right)$. We discuss the implications of this picture, sketched in Fig. 1, for the density of these various excitations, for their effect on plasticity and on lowtemperature properties of glasses as well as for the glass transition.

\section{GENERATING GAPPED GLASSES}

To generate ultrastable glasses displaying a finite gap, we follow a procedure similar to [35]. We consider breathing particles whose individual size can vary according to an energetic cost of characteristic stiffness $K$ (see Appendix B). The particles interact with a repulsive potential, up to a finite cutoff radius, chosen such that the potential remains continuous up to its third derivative [38] and thus allowing for a well-defined Hessian. At a given temperature, this system is known to be thermodynamically equivalent to a system of given (and continuous) polydispersity, and can be simulated using a usual molecular dynamics (MD). Including this
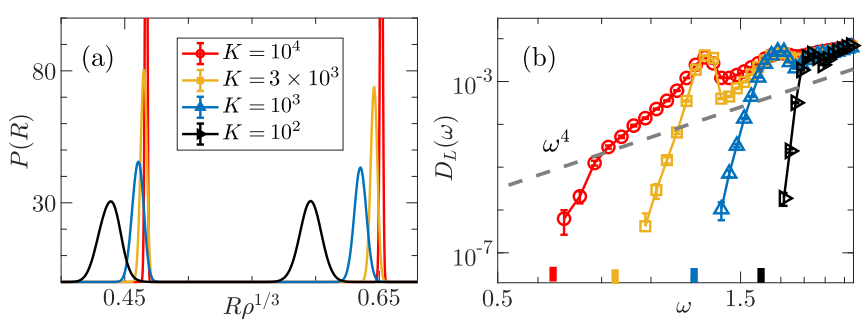

FIG. 2. (a) Distribution of particle radii, normalized by the number density $\rho=N /\langle V\rangle$, for different values of stiffness $K$ (the particle sizes are narrowly distributed when $K$ is large). (b) Density of quasilocalized modes displaying a finite gap $\omega_{c}$, in contrast to the usual pseudogap scaling $D_{L}(\omega) \sim \omega^{4}$ indicated with a dashed line. The gap values $\omega_{c} \approx\{1.64,1.19,0.85,0.65\}$ corresponding, respectively, to $K=\left\{10^{2}, 10^{3}, 3 \times 10^{3}, 10^{4}\right\}$ are indicated using ticks, following the same color code. Physically, decreasing $K$ results to a larger gap and thus a more stable glass, and is associated to a larger polydispersity.

breathing degrees of freedom leads to a giant shortening of the equilibration time, comparable to that of swap algorithms [37,39]. In practice, we perform MD with breathing particles for a long duration $t_{p}$ at a temperature $T_{p}(K)$, chosen such as to minimize the energy of the states eventually obtained (see Appendix C), before quenching using a FIRE algorithm [40] in which particles can still breathe.

The polydispersity obtained for various values of stiffness $K$ is shown in Fig. 2(a) for $N=8000$ particles, in three dimensions and at fixed pressure. Next, we freeze the radius of each particle, and compute the usual Hessian of the potential energy: its eigenvectors correspond to the vibrational modes of the glass, and its eigenvalues are denoted $\omega^{2}$ since they correspond directly to the frequencies of vibrational modes, as we take the particle mass to be unity. Showing that these states are gapped requires considerable statistics; in fact, we collect the spectra of $n=4000$ independent realizations (see Appendix A for a precise statement) and average them in order to obtain the density of vibrational modes $D(\omega)$. We emphasize that for the considered small system size, quasilocalized modes are already found below the first plane waves [20].

$D(\omega)$ turns out to display a gap: there are no quasilocalized modes below a finite frequency $\omega_{c}$. Since we find $\omega_{c}$ to be even higher than the frequencies of the first plane waves for $K=\left\{10^{2}, 10^{3}\right\}$ we manually remove them in order to measure the density of quasilocalized modes $D_{L}(\omega)$, as shown in Fig. 2(b). We extract $\omega_{c}$ by fitting a power law $D_{L}(\omega) \sim\left(\omega-\omega_{c}\right)^{\zeta}$, and obtain the values $\omega_{c}=$ $\{1.64,1.19,0.85,0.65\}$, for $K=\left\{10^{2}, 10^{3}, 3 \times 10^{3}, 10^{4}\right\}$, indicated with markers in Fig. 2(b). Note that if we consider instead the minimal frequency observed as an estimate for $\omega_{c}$, our conclusions below are not affected (see Appendix D).

\section{FILLING UP THE GAP VIA THERMAL ACTIVATION}

To test the robustness of gapped states to thermal fluctuations, we reheat our samples to a temperature $T_{a}$ and run MD simulations for a duration $t_{a}$, before applying an instantaneous quench to zero temperature. This procedure is sketched in Fig. 1 (and further detailed in Appendix B), and is entirely 

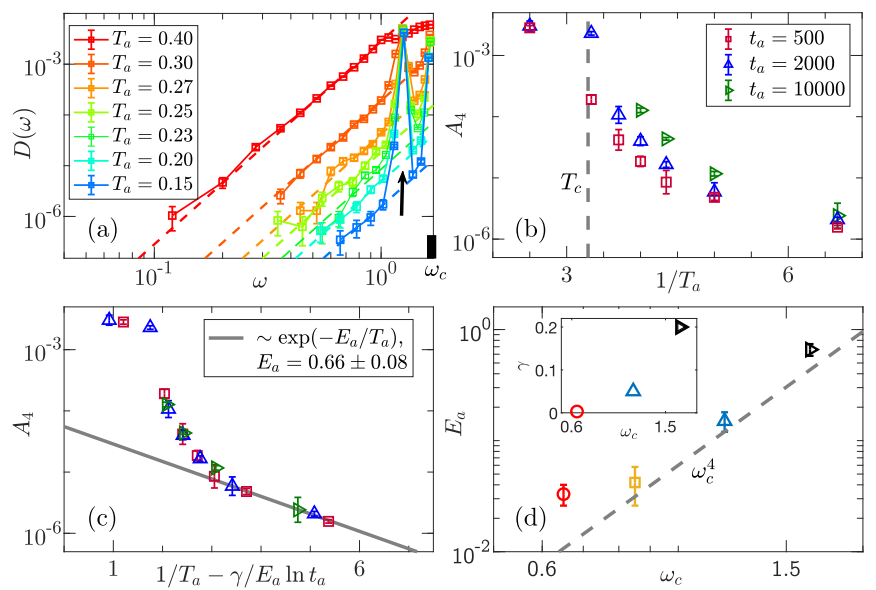

FIG. 3. (a) Density of soft modes $D(\omega)$ after reheating for a fixed duration $t_{a}=500$ at different temperatures $T_{a}$ (following the protocol sketched in Fig. 1), at fixed gap $\omega_{c}=1.64$ (the largest we generate, cf. Fig. 2). Note that the order of the legend matches the order of the curves. Furthermore, note that $D_{L}(\omega)=D(\omega)$ for $\omega<\omega_{e}$, with $\omega_{e}$ the frequency of the first plane wave, indicated with a black arrow. For reference, the mode-coupling temperature in this system is $T_{c} \approx 0.3$, and the gap, $\omega_{c}=1.64$, is indicated by a black tick. We emphasize that, before reheating, we had $D_{L}\left(\omega<\omega_{c}\right)=0$, so that the corresponding modes have been activated by thermal fluctuations. (b) Prefactor $A_{4}$ as a function of reheating temperature $T_{a}$ for different durations $t_{a}$. (c) Collapse of the different curves $A_{4}\left(T_{a}, t_{a}\right)$, supporting the functional form $A_{4}=f\left(t_{a}^{\gamma} \exp \left(-E_{a} / T_{a}\right)\right)$ where the function $f$ is linear at small argument, indicating an Arrhenius behavior at small $T_{a}$. (d) Typical energy scale $E_{a}$ vs the initial gap $\omega_{c}$, together with the associated scaling prediction $E_{a} \sim \omega_{c}^{4}$ (dashed line). Inset: Dynamical exponent $\gamma$ as a function of $\omega_{c}$.

performed at fixed particle radii. Upon reheating, local rearrangements [41] are thermally triggered (though less than $50 \%$ of the samples do rearrange at the lowest temperature that we probe [42]), consequently modifying the spectrum.

In Fig. 3(a) the low-frequency tail of $D(\omega)$ is shown for our most stable system (with $\omega_{c}=1.64$ ) for $t_{a}=500$ and varying $T_{a}$. Note that the acquisition of sufficient statistics required about $10^{5} \mathrm{CPU}$ hours. We always find that the gap is replaced by a pseudo-gap, compatible with the standard scaling:

$$
D_{L}(\omega) \approx A_{4}\left(T_{a}, t_{a}\right) \omega^{4}
$$

The prefactor $A_{4}$ characterises the density of quasilocalized excitations, and is extracted by fitting eq. (1) for $\omega<\omega_{e}$, where $\omega_{e}$ is the frequency of the first plane wave [see Fig. 2(b)]. As shown in Fig. 3(b), $A_{4}$ varies immensely (by three orders of magnitude), mostly due to the variation of the temperature $T_{a}$, with only a mild dependence on the time $t_{a}$. Moreover, we show in Fig. 3(c) that these curves can be collapsed, in the range of parameters probed, assuming the functional form $A_{4}\left(T_{a}, t_{a}\right)=f\left(t_{a}^{\gamma} \exp \left(-E_{a} / T_{a}\right)\right)$ and $\gamma=0.2$. The function $f$ is linear at small argument, supporting an Arrhenius behavior at low temperature $T_{a}$ (see Appendix D). Remarkably, this collapse indicates that for a given gap, the distribution of excitation energies is characterized by a single energy scale $E_{a}$ (presumably a lower cutoff, see below).
Interestingly, we find in Fig. 3(d) that $E_{a}$ very strongly increases with gap magnitude $\omega_{c}$ (see below for a proposed explanation). The dynamical exponent $\gamma\left(\omega_{c}\right)$ is also shown in the inset, and remains smaller than 0.2 in the entire range of initial gaps that we probe.

\section{MODES BEYOND THE GAP ACT AS AN EXCITATION RESERVOIR}

We saw that, if we start from a glass with an initially gapped density of states, thermal fluctuations will always populate this gap. To rationalize these findings, we consider the path of minimal energy connecting two states associated to one excitation, and denote by $s$ the curvilinear coordinate along it. The Taylor expansion of the energy along this path from the state 1 , by definition the one of minimal energy, reads

$$
E(s)=\frac{1}{2 !} \lambda_{1} s^{2}+\frac{1}{3 !} \kappa_{1} s^{3}+\frac{1}{4 !} \chi_{1} s^{4}+\mathcal{O}\left(s^{5}\right),
$$

which is a double-well, with a curvature $\lambda_{1} \approx \omega_{1}^{2}$ around the minima in state 1 . Physically, $\chi_{1}>0$ (otherwise the potential has no lower energy limit).

In that formalism, starting from a gapped glass corresponds to having a distribution $P\left(\lambda_{1}, \kappa_{1}, \chi_{1}\right)$ strictly zero at $\lambda_{1}<\omega_{c}^{2}$ and smooth above $\omega_{c}^{2}$. At finite temperature the gap is populated by thermal activation towards a state 2 with a smaller frequency $\omega_{2} \approx \sqrt{\lambda_{2}}$, which corresponds to a transition in an asymmetric double-well (as illustrated in Fig. 1). From Eq. (2) it is straightforward to obtain the expansion from state 2 , and the transformation $\left(\lambda_{2}, \kappa_{2}, \chi_{2}\right)=g\left(\lambda_{1}, \kappa_{1}, \chi_{1}\right)$. The joint distribution follows $P\left(\lambda_{2}, \kappa_{2}, \chi_{2}\right)=\left|g^{\prime}\left(\lambda_{2}, \kappa_{2}, \chi_{2}\right)\right| P\left(\lambda_{1}, \kappa_{1}, \chi_{1}\right)$ where the absolute value of the determinant of the Jacobian $\left|g^{\prime}\left(\lambda_{2}, \kappa_{2}, \chi_{2}\right)\right| \sim \lambda_{2}$ for small $\lambda_{2}$ (see Appendix E). Owing to the smoothness of $P\left(\lambda_{1}, \kappa_{1}, \chi_{1}\right)$ for $\lambda_{1} \gtrsim \omega_{c}^{2}$, for small $\lambda_{2}$ one has $P\left(\lambda_{2}, \kappa_{2}, \chi_{2}\right) \sim \lambda_{2}$ or equivalently $P\left(\omega_{2}, \kappa_{2}, \chi_{2}\right) \sim$ $\lambda_{2} d \lambda_{2} / d \omega \sim \omega_{2}^{3}$. After integrating on $\kappa_{2}$ and $\chi_{2}$ one gets $D_{L}\left(\omega_{2}\right) \sim \omega_{2}^{3}$. See Ref. [30] for a more general argument along the same line. Thus one expects to observe a pseudogap following thermally activated excitations. One effect will deplete the spectrum even further: in the case of a cubic pseudogap, the low-frequency spectrum is dominated by state 2 very close to a saddle node bifurcation (at the spinodal). However, once interactions among excitations are taken into account [43], configurations with such a large density of states near a saddle node bifurcation can be shown to be unstable and display avalanche-type events, i.e., where the relaxation of one excitation can destabilize others in turn [17]. This effect will increase the pseudogap exponent to values larger than three [44].

As far as the kinetics is concerned, the time scale $t_{a}$ on which an excitation equilibrates depends on the energy barrier $\Delta E$ to go from state 1 to 2 . It will occur (neglecting prefactors) when $t_{a} \gg t_{a}^{*} \sim \exp (\Delta E / T)$, i.e., its first-passage time. For much larger time scales, the probability of being in the excited states follows a Boltzmann factor $\exp \left(-E_{12} / T\right)$ at small $T$, where $E_{12}$ is the energy difference between the two states. If all states were equilibrated, $A_{4}$ would not depend on $t_{a}$ (i.e., $\gamma=0)$. By contrast, if no states were equilibrated $A_{4}$ would grow linearly in time. In that respect, our observation of the intermediate case $\gamma \approx 0.2$ is consistent with the notion that 

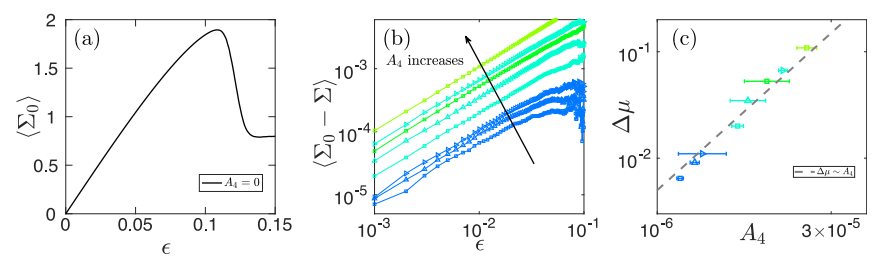

FIG. 4. (a) The ensemble averaged shear stress $\left\langle\Sigma_{0}\right\rangle$ as a function of strain $\epsilon$ for our largest gap $\left(\omega_{c}=1.64\right)$. (b) The difference $\left\langle\Sigma_{0}-\Sigma\right\rangle$ as a function of strain $\epsilon$ for different temperature cycles applied to the gapped glass from (a). As observed, the stress decreases as $A_{4}$ increases (from bottom to top, $T_{a}=$ $\{0.15,0.15,0.15,0.2,0.2,0.2,0.23,0.25\}$ corresponding to $t_{a}=$ $\left\{5 \times 10^{2}, 2 \times 10^{3}, 10^{4}, 5 \times 10^{2}, 2 \times 10^{3}, 10^{4}, 5 \times 10^{2}, 5 \times 10^{2}\right\}$, respectively). (c) The decrease in the effective shear modulus $\Delta \mu$ computed at $\epsilon=10^{-2}$ is proportional to $A_{4}$ : the dashed line corresponds to $\Delta \mu \sim A_{4}$.

there is a broad distribution of barriers, so that on the time scale $t_{a}$ a fraction of excitations are equilibrated, yet some barriers are still being jumped over for the first time.

For a given gap magnitude $\omega_{c}$, we expect to find a lower cutoff on the distribution of barriers $\Delta E$ (with the typical energy difference $E_{12}$ of the associated excitations being of the same order of magnitude). Consider for instance a symmetric double-well in the energy landscape and expand its energy around the maximum: $E(s)=-\frac{1}{2 !} \lambda s^{2}+\frac{1}{4 !} \chi s^{4}$. It is straightforward to show that in each minimum the frequency of the soft mode scales as $\sqrt{\lambda}$, allowing us to identify for the softest excitations $\lambda \sim \omega_{c}^{2}$. Likewise in this example the barrier for the double-well follows $\Delta E \sim \lambda^{2} \sim \omega_{c}^{4}$. This scaling holds for asymmetric double-wells as well (see Appendix E). Interestingly, our measured activation energy $E_{a}$ is compatible with this power-law relation, except for the smallest gap (hence less stable glass) that we study [45].

Overall, this analysis supports the scenario that modes beyond the gap act as a reservoir of excitations, with a broad distribution of barriers presenting a typical cutoff $E_{a} \sim \omega_{c}^{4}$ at low energies.

\section{EFFECTS OF A THERMALLY FILLED-UP GAP ON PHYSICAL PROPERTIES}

\section{A. The softening of loading curves is proportional to $\boldsymbol{A}_{4}$}

We now discuss the practical implications of the preparation dependent amplitude $A_{4}\left(T_{a}, t_{a}\right)$ on the mechanical properties of the ultrastable glass. The relationship between shear transformation and quasilocalized modes was studied in Ref. [17] for rapidly quenched glasses. More generally, more stably prepared systems exhibit a steeper loading curve and have a lower density of quasilocalized modes $[10,17]$. Here, we show a quantitative relationship between the amplitude of quasilocalized modes $A_{4}$ and the effective shear modulus during loading $\mu \equiv\langle\Sigma\rangle / \epsilon$, where $\epsilon$ is the imposed shear strain and $\langle\Sigma\rangle$ is the ensemble average of the corresponding shear stress increase [46]. More plasticity leads to a smaller $\mu$.

We measure the stress-strain response in ultra-stable glasses using a quasistatic loading protocol [see Fig. 4(a)]. We find that the gapped glasses have the highest effective shear modulus $\mu_{0}=\left\langle\Sigma_{0}\right\rangle / \epsilon$ and it decreases as the gap is filled and $A_{4}$ increases [Fig. 4(b)]. The reduction of the effective shear modulus $\Delta \mu\left(A_{4}\right) \equiv\left\langle\Sigma_{0}-\Sigma\left(A_{4}\right)\right\rangle / \epsilon$ is proportional to $A_{4}$ [see Fig. 4(c)] in the range of strains $\epsilon<0.01$ where it is strain independent.

\section{B. TLS disappear for large gaps}

We argue that TLS cannot be observed if a glass presents a large gap. Indeed if the tunneling amplitude is too small, on experimental timescales a single state is visited and TLS properties are not apparent [16]. It is precisely what happens when the gap is large, as barriers are then both larger and wider. To estimate this effect we follow the treatment of soft potential models [47] that solves the Schrödinger equation in potentials described by Eq. (2). For a symmetric double-well, the tunneling time follows $\tau=\hbar \pi / \Delta_{0}$, where $\Delta_{0}$ is the splitting energy stemming from quantum tunneling (and $\hbar$ is the reduced Planck constant). $\Delta_{0}$ is expressed as $\Delta_{0}=W \exp \left(-\left(\omega_{1} / \bar{\omega}\right)^{3}\right)$ where $W=\hbar\left(\hbar \chi_{1} /\left(96 m^{2}\right)\right)^{1 / 3}$ and $\bar{\omega}=\left(\hbar \chi_{1} /\left(2 m^{2}\right)\right)^{1 / 3}$ (cf. Ref. [47]), with $m$ the particle mass. Thus if $\omega_{1}>\omega_{c}^{*} \equiv\left(\ln \frac{\tau W}{\hbar \pi}\right)^{1 / 3} \bar{\omega}$, TLS are not apparent. In our simulations we find that the median of $\chi_{1} \approx 4.6 m \omega_{D}^{2} / a^{2}$ (for our largest gap) where $a$ is the interparticle distance and $\omega_{D}$ is the Debye frequency (see Appendix F for details). Taking estimates in amorphous silicon where $\omega_{D} \approx k_{B} 530 \mathrm{~K} / \hbar$ [48], we get $W \approx 0.03 \hbar \omega_{D}$ and $\omega_{0} \approx 0.1 \omega_{D}$. Considering the experimentally accessible time scale to be of order $\tau \approx 100 \mathrm{~s}$, at last we estimate $\omega_{c}^{*} \approx 0.3 \omega_{D}$. It is of the order of magnitude of our largest gap $\omega_{c} \approx 0.1 \omega_{D}$.

Suppressing TLS altogether would thus be accomplished by preparing sufficiently stable glasses so as to get $\omega_{c}>\omega_{c}^{*}$. These considerations stay valid even when thermal activation populates the gap and $A_{4}$ becomes finite, because quasilocalized modes with low-frequency correspond then to quite asymmetric wells whose barrier height and width (and therefore tunneling amplitude) is still comparable to the estimate above. The presence of a large underlying, thermally populated, gap of quasilocalized modes in ultrastable glasses thus offers an explanation for their lack of TLS $[13,14]$.

\section{REARRANGEMENTS INVOLVE FEWER PARTICLES AND BECOME STRINGLIKE AT LARGE GAPS}

We introduce an algorithm to decompose the displacement field of a rearrangement into several elementary excitations, which is needed to study how their geometry depends on the gap magnitude. Given a displacement field (induced, in our case, by the thermal cycle), we first consider the particle with maximal displacement, and draw a sphere of radius $\tilde{R}$ around it. Beyond this sphere, all the particle displacements are set to zero (i.e., these particles are set back to their initial position in the gapped state), whereas within the sphere the displacements are preserved. Next, we perform with that initial condition a steepest descent of the interaction energy. We find that if $\tilde{R}$ is small, all displacements go back to zero, whereas if $\tilde{R}$ is large, they do not. We consider the smallest $\tilde{R}$ of the latter case, and the displacement field obtained at the end of the corresponding gradient descent defines our first elementary excitation. Next, 

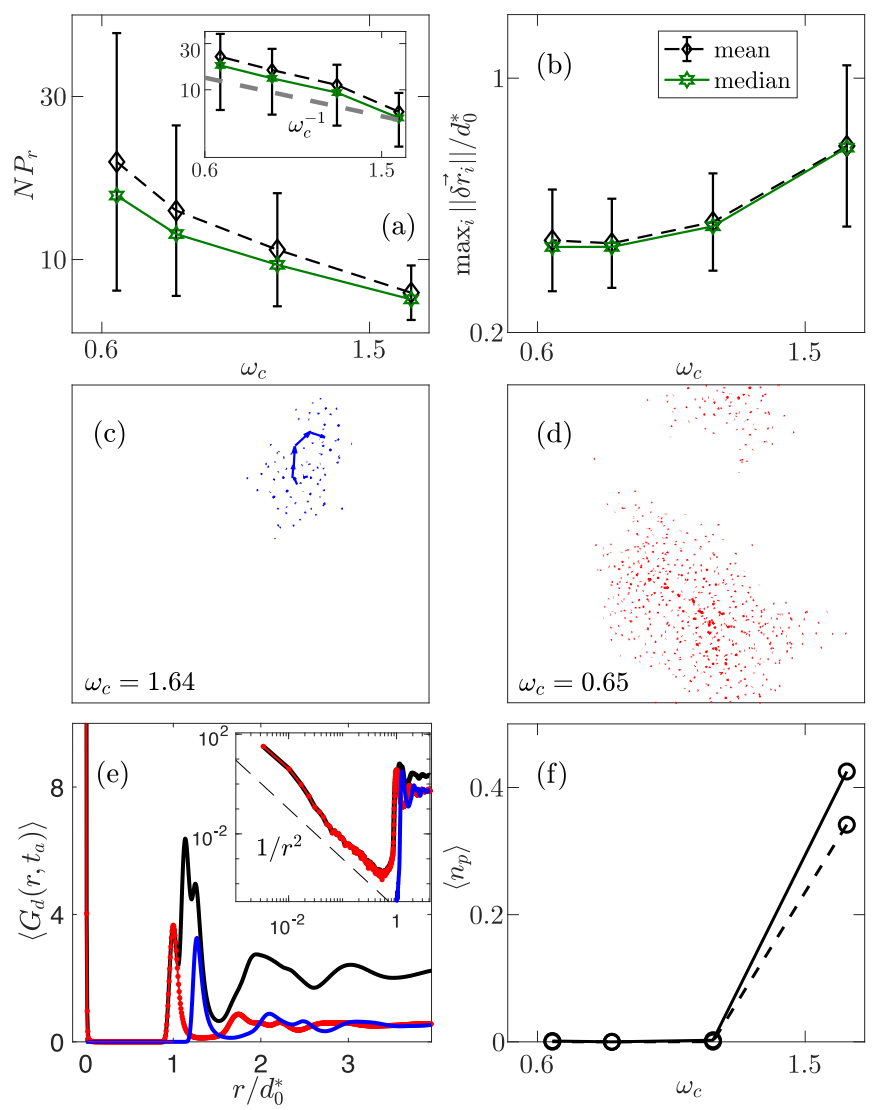

FIG. 5. (a) Mean (dashed black) and median (green) of the number of particles $N P_{r}$ involved in an individual excitation $v s \omega_{c}$ at $t_{a}=500$ (at the lowest temperature we probe for each gap). The error bars stand for the standard deviation of $N P_{r}$ which indicates that the distribution of $N P_{r}$ is broader for smaller $\omega_{c}$. (b) Mean (dashed black) and median (green) of the displacement norm of the most mobile particle in an excitation, as a function of $\omega_{c} . d_{0}^{*}$ is the most frequent diameter of the smaller particles $\left(d_{0}^{*}=\{0.738,0.918,0.963,0.988\}\right.$ for the different $\left.\omega_{c}\right)$. (c),(d) Thermally induced rearrangement, respectively for our largest $\left(\omega_{c}=1.64\right)$ and smallest $\left(\omega_{c}=0.65\right)$ gaps, projected on the $x y$ plane. (e) Ensemble and radially averaged (on all observed excitations) Van Hove correlation function $G_{d}\left(r, t_{a}\right)$ computed for all (in black and having largest $G_{d}$ at large $r$ ), only small (red dotted) or only large (blue) particles for $\omega_{c}=1.64$. The peak around $r=0$ corresponds to permutations of particles. The black and red curves overlap, indicating that permutations only occur on small particles. (f) Average number of permutations $\left\langle n_{p}\right\rangle v s \omega_{c}$, for two different cutoff distances $r_{c} / d_{0}^{*}=\{0.025,0.05\}$ (dashed and solid, respectively).

we subtract this obtained displacement field from the full one, and repeat the entire procedure recursively until no more excitations are found (see Appendix G for details and visual examples).

Given an individual excitation of displacement field $\left\{\delta \vec{r}_{i}\right\}$, we compute, from its associated participation ratio, an estimate of the number of particles involved in this excitation $N P_{r} \equiv\left[\sum_{i}\left\|\delta \vec{r}_{i}\right\|^{2}\right]^{2} / \sum_{i}\left\|\delta \vec{r}_{i}\right\|^{4}$. For each gap magnitude $\omega_{c}$, we find about 5000 such excitations and report the mean and the median of this observable in Fig. 5(a). We find that the typical number of particles involved in one excitation decreases as $\omega_{c}$ increases. We propose the following rationalisation. The length scale of quasilocalized modes was found to be proportional to the characteristic length scale entering the response to a local dipole [23], as proposed based on a variational argument in Ref. [49]. The length scale $\ell_{c}$ entering the dipolar response was observed to decrease as the system moves away from a marginally stable phase and enters a gapped solid phase as $\ell_{c} \sim 1 / \sqrt{\omega_{c}}$ [50]. The volume of the corresponding mode was shown to go as $\ell_{c}^{2}$ (independent of the number of dimensions) for elastic networks of springs at rest [49,50]. Taken together, these results correspond to a number of particles involved in an excitation that decreases with increasing gap as $N P_{r} \sim 1 / \omega_{c}$. As shown in the inset of Fig. 5(a), this is in reasonable agreement with our observations.

A complementary observable for the geometry is the maximum displacement $\max _{i}\left\{\left\|\delta \vec{r}_{i}\right\|\right\}$ for a given excitation, whose mean and median values for all excitations at a given $\omega_{c}$ are shown in Fig. 5(b). Interestingly, this maximum displacement increases with the gap, and becomes close to the small particle diameter equal to $d_{0}^{*}$.

A direct visualization of the excitation fields reveals a (presumably related) interesting phenomenon: for our largest gap, the displacements are stringlike with several particles exchanging positions, as shown in Fig. 5(c), whereas for smaller gaps they are much more compact and no permutations occur. To quantify this effect, we follow the glass transition literature [4] and measure the distinct part of the Van Hove correlation:

$$
G_{d}(\vec{r}, t) \equiv \frac{1}{N}\left\langle\sum_{i=1}^{N} \sum_{j(\neq i)}^{N} \delta\left(\vec{r}-\vec{r}_{j}(t)+\vec{r}_{i}(0)\right)\right\rangle,
$$

where the average is made on all the observed elementary excitations at some given $\omega_{c}$. It is plotted for our most stable system in Fig. 5(e) after radially averaging. The key observation is the presence of a very sharp peak around $r=0$, which can only arise from particles replacing each other. Interestingly, if we condition our definition of the Van Hove correlation to large or small particles only, we find that the peak only persists for small particles [in red in Fig. 5(e)]. Strings thus correspond to smaller particles navigating in an environment of larger ones.

Next we integrate the peak around $r=0$ to quantify the number of permuting particles averaged on all elementary excitations:

$$
\left\langle n_{p}\right\rangle=\left\langle N \int_{0}^{r_{c}} G_{d}\left(r, t_{a}\right) 4 \pi r^{2} d r\right\rangle,
$$

where $r_{c}$ is a cutoff that is tuned. We observe that permutations are essentially absent except for the largest considered gap, see Fig. 5(f).

\section{DISCUSSION}

In summary, we have argued that in gapped glasses, modes beyond the gap act as an excitation reservoir for thermal activation. This effect always destroys the gap and leads to a density of quasilocalized modes $D_{L}(\omega) \approx A_{4}(T) \omega^{4}$. At low temperatures, we found that $A_{4} \sim \exp \left(-E_{a}\left(\omega_{c}\right) / T\right)$ where the typical energy scale $E_{a}\left(\omega_{c}\right)$ is a rapidly increasing function of $\omega_{c}$. A simple scaling prediction gives $E_{a} \sim \omega_{c}^{4}$, in good 
agreement with our observations, except for the smallest gap value that we explore. Finally, we observed that as the gap increases, excitations involve fewer and fewer particles and become more and more stringlike. The growing length scale of the excitations as $\omega_{c} \rightarrow 0$ is consistent with the previously identified growing length characterising the elastic response of an amorphous solid near a macroscopic elastic instability [50].

Although our observations were made in ultrastable states obtained by a specific protocol [51], our arguments on this reservoir effect are much more general. Assuming that this effect is at play in supercooled liquids ties together several unexplained observations, as we now discuss.

Reinterpreting mean-field descriptions of glasses. Goldstein [52] proposed early on that the glass transition takes place near some temperature $T_{c}$ below which most normal modes become stable. Such an enhanced stability is consistent with the overall elastic stiffening upon cooling apparent in the bulk [53] or local [22] elastic moduli in fragile supercooled liquids. Theoretically, this view is consistent with mean-field models of the glass transition in infinite dimensions - that are closely related to mode-coupling theory - [32,33], in which the spectrum of the Hessian becomes stable and opens a gap with $\omega_{c}^{2} \sim\left(T_{c}-T\right)$ [34]. Our work suggests a natural way to extend this picture to finite dimensions as sketched in Fig. 1: the gap is decorated by excitations stemming from the reservoir of modes with $\omega \geqslant \omega_{c}$. In this approach (i) the excitation density strongly decreases with temperature: away from $T_{c}$ in the deeply supercooled regime, it should be proportional to $\exp \left(-E_{a}\left(\omega_{c}(T)\right) / T\right)$ and (ii) as $T$ decreases, $\omega_{c}$ increases and excitations are less and less extended. Point (i) offers an explanation for the very rapid decay upon cooling of $A_{4}(T)$ [25,27], TLS density [28] and shear transformations $[10,54]$ observed in ultrastable supercooled liquids. Point (ii) is consistent with the result that TLS [28] and quasilocalized modes [25,27] present a lower participation ratio upon cooling (such changes of geometry may lead to additional effects on their density [55] ).

Glass transition. The mean-field proposal that supercooled liquids present an effective gap growing upon cooling, leading to a rarefaction of thermally accessible excitations, is consistent with the observation that rearrangements become stringlike with more and more particles exchanging positions upon cooling [4,5], since we find that excitations at the large gap are precisely like that. In our view, why elementary excitations display such a geometry at large gap is yet to be explained [56].

At our lowest temperatures, only one or a few strings get activated, which can only lead to a very partial relaxation of the system. Isolated strings thus belong to the class of $\beta$ relaxation in supercooled liquids, as shown in Ref. [5], using normal dynamics, for model metallic glasses (molecular and covalent liquids may certainly present other $\beta$-relaxation mechanisms governed by the local chemistry, such as dangling bonds). Yet strings may also contribute to the $\alpha$ relaxation of liquids if they are present in sufficient density, at least for the continuously polydisperse ones receiving much attention currently. Indeed they allow for the exchange of particles with distinct radii. Such swap moves are now known to relax the system with great efficiency, so the dynamics should not be slower than the time scale to naturally operate these swaps $[57,58]$. The rapid increase of their characteristic low-energy cutoff $E_{a}$ with growing gap would then contribute to the fragility of liquids.

Note that such views in which activation deep in the supercooled liquid phase is controlled by $T_{c}$, contrasts with the usual interpretation of mean-field results in which activation is controlled by an entropy crisis occurring at a lower temperature $T_{K}$ (the Kauzmann temperature) [32,33]. The latter is in our opinion ruled out in poly-disperse systems by the recent observation that changes of kinetic rules (such as allowing for swap moves [59]) immensely affect the location of the glass transition, while leaving intact thermodynamic properties [57]. Changes of kinetic rules, however, affect the location of the mode coupling temperature $T_{c}$ [37,58], thus pictures of activation based on that temperature are consistent with the observations of swap algorithms.

Effects of rare fluctuations. We have shown that a gap in the density of quasilocalized modes cannot exist in finite dimension at finite temperature, due to the thermal activation of their associated excitations. We expect that at least another effect will enter in finite dimensions to fill up the gap. In electronic systems that present impurities, the density of states does not vanish in the range of energies where the pure system would, due to rare regions where many impurities are present. This effect leads to the so-called "Lifshitz tail" in the electronic density [60]. In glasses, we expect that aspects of the structure controlling stability, such as coordination and pressure $[61,62]$, will also fluctuate and lead to rare weaker regions in the materials (we do not see this effect in our breathing particles, whose preparation may lead to an unusually homogeneous material). Such fluctuations will need to be larger and larger as the gap grows to contribute to low-frequency quasilocalized modes, and therefore less likely, leading to a rapidly decaying density of quasilocalized modes with growing gap. These atypical rare regions may have little effect for plasticity or structural relaxation near the glass transition, but may be important in affecting the density of TLS. It would thus be interesting in the future to study glasses of controlled inhomogeneity to separate rare fluctuations in the structural disorder from the excitations reservoir effect introduced here.

\section{ACKNOWLEDGMENTS}

We thank L. Berthier, G. Biroli, C. Cammarota, D. Khomenko, C. Liu, V. Lubchenko, M. Müller, M. Ozawa, C. Scalliet, P. Wolynes, and F. Zamponi for fruitful discussions, as well as G. Kapteijns and E. Lerner for discussions and numerical support at the beginning. T.W.J.d.G. acknowledges support from The Netherlands Organisation for Scientific Research (NWO) by a NWO Rubicon Grant 680-50-1520 and from the Swiss National Science Foundation (SNSF) by the SNSF Ambizione Grant No. PZ00P2_185843. E.A. acknowledges support from the SNSF by the SNSF Ambizione Grant No. PZ00P2_173962, and M.W. by the Simons Foundation Grant (No. Matthieu Wyart) and from the SNSF under Grant No. 200021-165509. 
TABLE I. Parameters and key quantities in the simulation.

\begin{tabular}{lcccc}
\hline \hline$K$ & $10^{2}$ & $10^{3}$ & $3 \times 10^{3}$ & $10^{4}$ \\
\hline$\omega_{c}$ & 1.65 & 1.19 & 0.85 & 0.65 \\
$n$ & 4000 & 4000 & 2000 & 3000 \\
$N$ & 8000 & 8000 & 8000 & 8000 \\
$\phi$ & 0.62 & 0.74 & 0.77 & 0.79 \\
$\langle V\rangle$ & $3722.0 \pm 0.3$ & $7303.9 \pm 0.7$ & $8607.2 \pm 0.9$ & 0.9 \\
$\rho$ & 2.15 & 1.10 & 0.44 & 0.86 \\
$T_{p}$ & 0.2 & 0.39 & $1 e 4$ & 0.49 \\
$t_{p}$ & $1 e 4$ & $1 e 4$ & 0.525 & $1 e 4$ \\
$T_{c}$ & 0.304 & 0.484 & $\{0.03,0.05,0.1\}$ & 0.572 \\
$T_{a}$ & $\{0.15,0.20,0.23$, & $0.07,0.10,0.20,0.30$, & $\{0.03,0.05,0.10,0.20$, \\
$t_{a}$ & $0.25,0.27,0.30,0.40\}$ & $0.39,0.50,0.60,0.80\}$ & $\{500\}$ & $0.30,0.49,0.60,0.80\}$ \\
$E_{a}$ & $\{500,2000,10000\}$ & $0.100,500,2000\}$ & $0.042 \pm 0.016$ & $\{100,500,2000\}$ \\
$\gamma$ & $0.66 \pm 0.08$ & $0.15 \pm 0.03$ & - & $0.03 \pm 0.007$ \\
$\left\langle n_{e}\right\rangle$ & 0.2 & 0.05 & 1.2 & 0.003 \\
\hline \hline
\end{tabular}

\section{APPENDIX A: PARAMETERS AND KEY QUANTITIES}

Table I shows the parameters and key quantities in our simulation. Notice that $\phi \equiv \sum_{i=1}^{N} \frac{4}{3} \pi R_{i}^{3} / V$ is the packing fraction; and that $\langle V\rangle$ is the ensemble average volume (and the uncertainty its standard deviation). The average number of excitations per realization $\left\langle n_{e}\right\rangle$ has been obtained with $t_{a}=500$ at the lowest $T_{a}$ we probed for each $K . T_{c}$ is obtained from the relation: relaxation time $\sim\left(T-T_{c}\right)^{-v}$ [31], where $v$ is also a fit parameter. Lengths $\left(\vec{r}, R_{i}\right.$ and $\left.L\right)$ are shown in the unit of $d_{0}$ (or the most frequent diameter of small particles $d_{0}^{*}$, if so indicated): the diameter of an initially small particle. Energies $\left(E_{a}\right)$ are expressed in the unit of $\varepsilon$ that is the prefactor of the pair interaction potential (see below). Temperature $\left(T_{p}\right.$, $T_{a}$, and $\left.T_{c}\right)$ is in the unit of $\varepsilon / k_{B}$. Time $\left(t_{p}, t_{a}\right.$, and $\left.\omega^{-1}\right)$ is shown in the unit of $t_{0}$ where $t_{0} \equiv \sqrt{m d_{0}^{2} / \varepsilon}$.

\section{Detecting a rearrangement}

To detect if reheating with a temperature $T_{a}$ for a duration of $t_{a}$ has led to a rearrangement we consider the ratio of the norm and the participation ratio of the displacement field:

$$
\mathcal{I} \equiv \frac{\left\|\delta \vec{r}_{i}\right\|}{P_{r}\left(\delta \vec{r}_{i}\right)}
$$

where $\left\|\delta \vec{r}_{i}\right\|$ is the Euclidean norm of the particle displacement field, $\delta \vec{r}_{i}$, between the quenched states before and after reheating, and its participation ratio

$$
P_{r}\left(\delta \vec{r}_{i}\right) \equiv \frac{\left(\sum_{i}\left\|\delta \vec{r}_{i}\right\|^{2}\right)^{2}}{N \sum_{i}\left\|\delta \vec{r}_{i}\right\|^{4}} .
$$

If a rearrangement results from reheating, the norm of the displacement field is finite (typically $10^{-3}-10^{-2}$ ) and participation ratio is on the order of $10^{-3}-10^{-2}$. In contrast, if there was no rearrangement, the norm of the displacement field is of the order of the numerical precision $\left(10^{-6}\right)$ and the participation ratio is of order one. We distinguish the two cases using a threshold. We define that a rearrangement has taken place if $\mathcal{I}>10^{-3}$. Note that the distributions of $\mathcal{I}$ corresponding to the two cases are clearly separated.

\section{APPENDIX B: MOLECULAR DYNAMICS}

1. Sample preparation: 'breathing' dynamics

We study a three-dimensional periodic particle system of $N=8000$ particles, that is characterized by the grand potential

$$
\mathcal{U}=\sum_{i<j} \varphi\left(r_{i j}, R_{i}, R_{j}\right)+\sum_{i} \mu\left(R_{i}, R_{i}^{(0)}\right)
$$

where $\varphi$ is a purely repulsive inverse power-law potential, defined

$$
\begin{aligned}
\varphi & \left(r_{i j}, R_{i}, R_{j}\right) \\
\quad & = \begin{cases}\varepsilon\left[\left(\frac{R_{i j}}{r_{i j}}\right)^{10}+\sum_{p=0}^{3} c_{2 p}\left(\frac{r_{i j}}{R_{i j}}\right)^{2 p}\right], & \frac{r_{i j}}{R_{i j}} \leqslant r_{c} \\
0, & \frac{r_{i j}}{R_{i j}}>r_{c}\end{cases}
\end{aligned}
$$

with $r_{c}$ is the cutoff distance, $R_{i j} \equiv R_{i}+R_{j}$ (two times the average particle radius), and $r_{i j} \equiv\left\|\vec{r}_{i j}\right\| \equiv\left\|\vec{r}_{i}-\vec{r}_{j}\right\|$ (the Euclidean norm of the distance vector separating particles $i$ and $j$ ). $c_{2 p}$ is a constant that makes $\varphi$ continuous up to the third derivative at $r_{c}$. Furthermore,

$$
\mu\left(R_{i}, R_{i}^{(0)}\right)=\frac{K}{2}\left(1-\frac{R_{i}^{(0)}}{R_{i}}\right)^{2}\left(R_{i}^{(0)}\right)^{2}
$$

is a chemical potential that allows a particle to change its size from its initial value $R_{i}^{(0)}$ at an energetic cost that scales with a modulus $K$. For $K=\infty$ it is impossible for a particle to change its radius, while it becomes easier as $K \rightarrow 0$. The initial particle radii are bidisperse, in a 50:50 mixture. In particular, in one randomly selected, half of the particles has $R_{i}^{(0)}=0.5 d_{0}$ and the other half has $R_{i}^{(0)}=0.7 d_{0}$ (where $d_{0}$ sets the unit of length of our system).

Sample preparation proceeds by instantaneously heating the initial random configuration to a temperature $T_{p}$ and keeping it at this temperature for a certain time $t_{p}$ under the constraint of a fixed pressure $p=20.0$ (in units of $\varepsilon / d_{0}^{3}$ ). We then instantaneously quench the system to zero temperature 

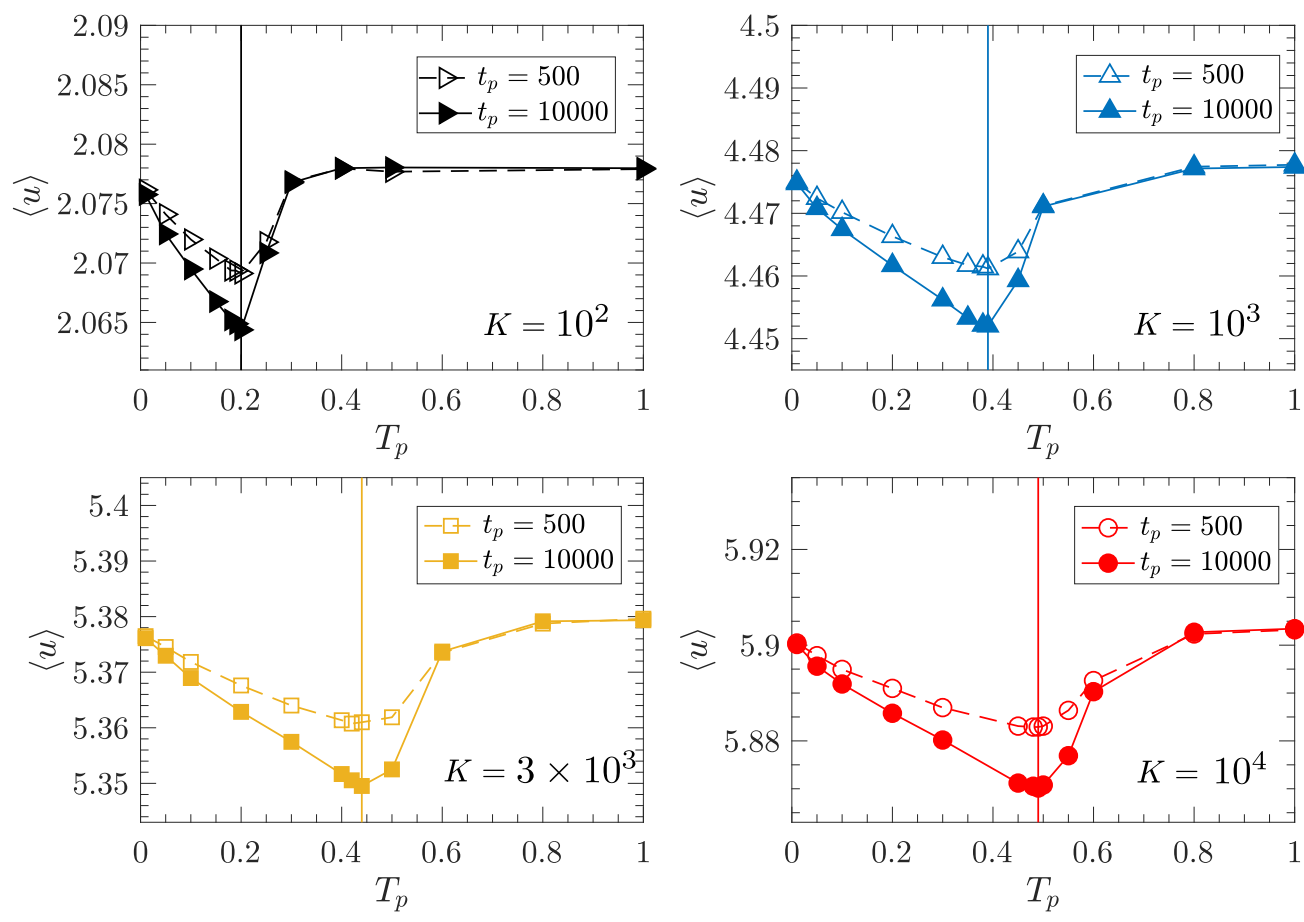

FIG. 6. Mean interaction potential energy $\langle u\rangle$ after sample preparation with breathing dynamics for varying parent temperature $T_{p}$ and two different waiting times $t_{p}$. The different panels correspond to different $K$ as indicated. The selected temperature $T_{p}$ for which the potential energy is lowest for the largest practically reachable $t_{p}=10^{4}$ is indicated using vertical lines (see Appendix A for numeric values).

by minimising the grand potential. See algorithmic details below.

\section{Activation by temperature: normal dynamics}

We proceed by fixing the particle size, which corresponds to a potential energy

$$
U=\sum_{i<j} \varphi\left(r_{i j}, R_{i}, R_{j}\right)
$$

(see Eq. (B2) for the definition of $\varphi$ ). We then gently heat the system configuration to a certain "activation temperature" $T_{a}$ [at a heating rate $T_{a} /\left(10 t_{0}\right)$ ], and keep the sample at $T_{a}$ for a total duration $t_{a}$. Thereafter, we instantaneously quench the sample to zero temperature. Algorithmic details are listed below.

\section{Molecular dynamics algorithm}

We run molecular dynamics, whereby the particle dynamics are given by Newton's equation of motion with the gradient of the potential energy on a particle as driving force. Time is discretized in steps of $\Delta t$ using the standard velocity Verlet algorithm. The temperature and pressure are controlled using a Berendsen thermostat [68], where the temperature is defined as the total kinetic energy $\sum_{i} m|| \dot{\vec{r}}_{i} \|^{2} / 2$ (where $\bullet$ refers to the time derivative). Note that during preparation the kinetic energy is $\sum_{i}\left(m\left\|\dot{\vec{r}}_{i}\right\|^{2}+\dot{R}_{i}^{2}\right) / 2$. We use the FIRE algorithm [40] to quench the systems.

For completeness we report that $r_{c}=1.48 d_{0}, \varepsilon=1$, $m=1, \quad d_{0}=1, \quad$ and $\Delta t=0.005$. Furthermore $c_{0}=$
$-1.1106337662511798, \quad c_{2}=1.2676152372297065, \quad c_{4}=$ $-0.4960406072849212, c_{6}=0.0660511826415732$; see the Supplemental Material of Ref. [38].

\section{APPENDIX C: SAMPLE PREPARATION}

We choose $T_{p}$ and $t_{p}$ to empirically generate a configuration in the lowest possible energetic state in terms of the mean interaction energy $\langle u\rangle=\langle U\rangle / N$ (averaged on an ensemble of $n=10$ samples). In particular, we set $t_{p}=10^{4}$ (the highest value we can practically reach, with each sample taking eight CPU hours to prepare). We manually optimize $T_{p}$ as reported in Fig. 6. Note that we verify that the $T_{p}$ at which we find the optimum, is robust in terms of preparation duration $t_{p}$, by comparing our results to those for $t_{p}=500$ (dashed line in Fig. 6). Furthermore, the reader is reminded that although the particle size distribution depends on temperature while still at $T_{p}$, the final particle size distribution at zero temperature is independent of $T_{p}$. Note that our breathing dynamics (at small $K$ ) are quite efficient to prepare samples in a low potential energy state. We verify this by preparing an ensemble (again $n=10$, but with $N=2000$ particles) with normal dynamics and a slow quench rate. We plot the potential energy $\langle u\rangle$ at different temperatures in Fig. 7. In all cases $\langle u\rangle$ at $T=0$ is higher than that for the sample prepared using breathing dynamics, which was prepared at a fraction of the computational costs (sample preparation is a factor of 2000 faster using breathing dynamics). 


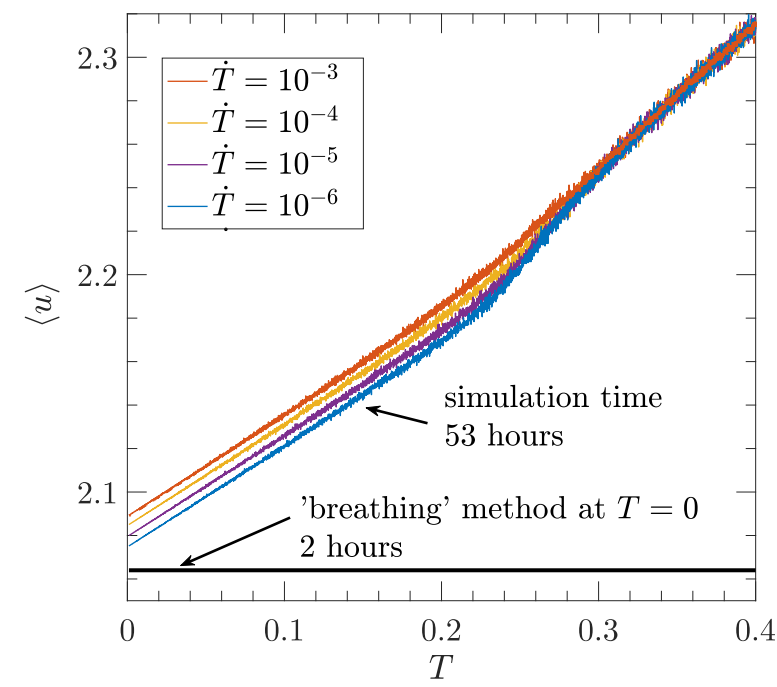

FIG. 7. Mean interaction potential energy as obtained by sample preparation using breathing dynamics (at $T=0$ for $K=10^{2}, t_{p}=$ $10^{4}$, and $T_{p}=0.2$, in black) and using normal dynamics at different cooling rates (cooling from $T=0.4$ ) as indicated in the legend. Note that in both cases the ensemble comprises $n=10$ samples, but that normal dynamics are run using smaller than usual samples comprising $N=2000$ particles ( $N=8000$ is used throughout). We verify the representativeness of these smaller samples using $N=8000$ for $\dot{T}=10^{-3}$, shown using a dashed red line (that indeed coincides with the solid red line for $N=2000$ ). The required CPU time to run the entire simulation with $N=2000$ particles is indicated. From bottom to top, $\dot{T}$ increases. Note that for our breathing dynamics the time has been divided by four to correct for the difference in system size.

\section{APPENDIX D: MEASUREMENT OF QUASILOCALIZED MODES}

\section{Spectrum of the Hessian}

We extract the Hessian (or stiffness matrix) - the second derivative of interaction energy - as follows:

$\mathcal{H}_{i j} \equiv \frac{\partial^{2} U}{\partial \vec{r}_{i} \partial \vec{r}_{j}}=-\frac{d^{2} \varphi\left(r_{i j}\right)}{d r_{i j}^{2}} \frac{\vec{r}_{i j} \vec{r}_{i j}}{r_{i j}^{2}}-\frac{d \varphi\left(r_{i j}\right)}{d r_{i j}} \frac{1}{r_{i j}}\left(\mathcal{I}-\frac{\vec{r}_{i j} \vec{r}_{i j}}{r_{i j}^{2}}\right)$

for $i \neq j$. The diagonal

$$
\mathcal{H}_{i i}=-\sum_{i \neq j} \mathcal{H}_{i j}
$$

due to translation symmetry. Note that $\mathcal{H}_{i j}$ is a second-order tensor, and that $\mathcal{I}=\delta_{\alpha \beta} \vec{e}_{\alpha} \vec{e}_{\beta}$ is a second-order unit tensor. We then diagonalize the Hessian, leading to $N$ eigenvalues $\lambda$ and corresponding eigenmodes $\vec{\Phi}_{i}$. Because all particles have a mass $m=1$ the corresponding $N$ eigenfrequencies are

$$
\omega \equiv \sqrt{\lambda}
$$

We finally represent the spectrum of the Hessian as

$$
D(\omega)=\frac{1}{3 N-3} \sum_{k=1}^{3 N-3} \delta\left(\omega-\omega_{k}\right)
$$

\section{Density of quasilocalized modes}

The density of quasilocalized modes, $D_{L}(\omega)$, follows from the spectrum of the Hessian in Eq. (D4) by filtering plane waves that have a frequency $\omega_{e}<\omega_{c}$ (where $\omega_{c}$ is defined below). We identify these plane waves by their signature in participation ratio

$$
P_{r}\left(\vec{\Phi}_{i}\right) \equiv \frac{\left(\sum_{i}\left\|\vec{\Phi}_{i}\right\|^{2}\right)^{2}}{N \sum_{i}\left\|\vec{\Phi}_{i}\right\|^{4}} .
$$

Plane waves thereby have $P_{r} \approx 2 / 3$, while quasilocalized modes have $P_{r} \ll 1$.

In practice, most of our samples have no plane waves below $\omega_{c}$, rendering filtering obsolete. In fact, we only apply filtering after sample preparation for $K=\left\{10^{2}, 10^{3}\right\}$. Since we empirically observe the plane waves to be well separated from the quasilocalized modes in terms of frequency, we remove them by removing the first $3+12$ eigenmodes of each realization for $K=10^{3}$ and $3+12+24$ eigenmodes of each realization for $K=10^{2}$, corresponding to the three translational modes and the first (two) bands of plane waves [69]. Note that $D_{L}(\omega)$ is not renormalized after filtering of plane waves.

We emphasize that in all other measurements $D_{L}(\omega)=$ $D(\omega)$ at low frequency.

\section{Protocol to measure $\omega_{c}$}

We measure the gap frequency $\omega_{c}-$ the frequency of the first quasilocalized mode. To measure $\omega_{c}$, we assert that the density of soft quasilocalized modes follows

$$
D_{L}(\omega) \sim\left(\omega-\omega_{c}\right)^{\zeta}
$$

at low frequency $\omega$. We then move $\omega_{c}$ until the power law is most obvious at low $\omega$, as shown in Fig. 8. We then visually extract the power $\zeta$ and check that it and the extracted $\omega_{c}$ are consistent with extreme-value statistics. In particular, we expect

$$
\omega_{\min }^{\prime}-\omega_{c} \sim\left(n^{\prime}\right)^{-1 /(1+\zeta)}
$$

where $\omega_{\min }^{\prime}$ is the frequency of the softest quasilocalized mode in an ensemble of $n^{\prime}$ realisations chosen as a random subset of our ensemble of $n$ realisations. We consider $\bar{\omega}_{\text {min }}^{\prime}$ the average of lowest three realisations (out of $n^{\prime}$ realisations). Indeed, our extracted $\omega_{c}$ is consistent with this scaling, as shown in the insets of Fig. 8. In addition, we check that $\omega_{c}$ is robust to a change of system size (shown in Fig. 6).

\section{Protocol to fit $\boldsymbol{A}_{4}$}

$A_{4}$ is extracted from $D_{L}(\omega)$ by fitting

$$
D_{L}(\omega)=A_{4} \omega^{4}
$$

(i.e., Eq. (1)) for frequencies below the first plane wave (for $K=10^{2}$ ) and for frequencies below $\omega_{c}$ (for $K=10^{3}$, $3 \times 10^{3}$, and $\left.10^{4}\right)$. Note that consequently $D_{L}(\omega)=D(\omega)$ in the relevant frequency range for all these measurements. The mean and the error of $A_{4}$ follow as the mean and standard deviation of $\left\{\ln D\left(\omega_{i}\right)-4 \ln \omega_{i}\right\}$ where $\omega_{i}$ corresponds to the position of the bins of $D(\omega)$.

We verify that the value of $A_{4}$ that we fit is robust to a mild decrease of system size (using $n=2000$ realizations of 

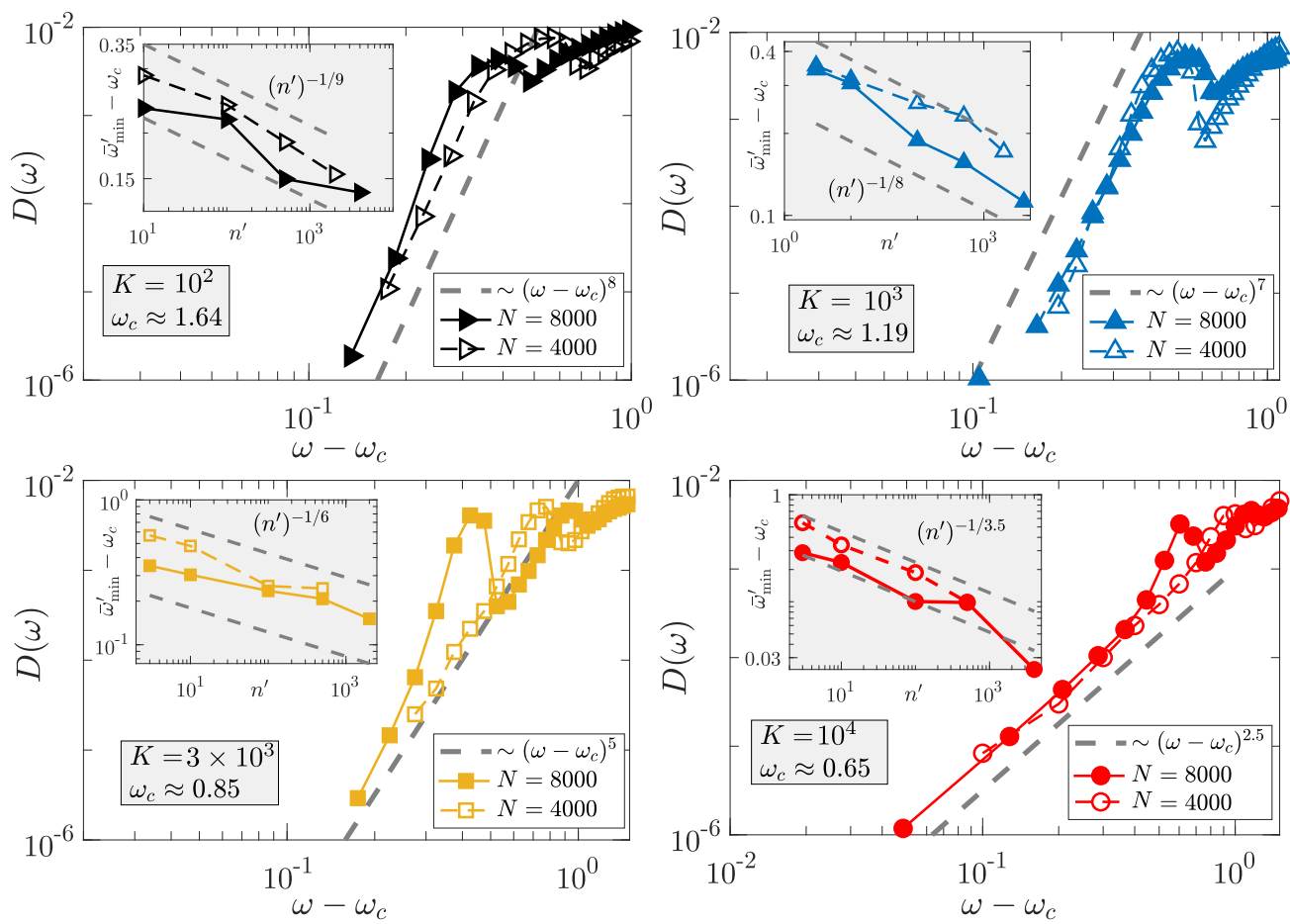

FIG. 8. Fit of $\omega_{c}$ by asserting the power law scaling in Eq. (D6) and check by extreme value statistics as in Eq. (D7) (insets), for all considered $K$.

$N=4000$ particles, compared to an ensemble of $n=4000$ realizations of $N=8000$ particles). We find that both the density of soft quasilocalized modes and the extracted $A_{4}$ are robust to the change of system size, as reported in Fig. 9.

\section{Protocol to fit $E_{a}$ and $\gamma$}

Our protocol to fit $E_{a}$ and extract $\gamma$ consists of two steps. 1) We first collapse the curves of $A_{4}\left(T_{a}\right)$ for different $t_{a}$. Thereto we shift the horizontal axis of, e.g., Fig. 3(b) in accordance with assuming a functional dependence

$$
A_{4}=A_{4}\left(t_{a}^{\gamma} e^{-E_{a} / T_{a}}\right),
$$

until the curves for different $t_{a}$ collapse to a single curve [e.g., Fig. 3(c)], by optimising the ratio $\gamma / E_{a}$. 2) On the master curve we next fit $E_{a}$ of low $T_{a}$. Since we know the ratio $\gamma / E_{a}$, the fitted value of $E_{a}$ gives us direct access to $E_{a}$. Specifically, we fit $\ln \left(A_{4}\right)$ vs $1 / T_{a}-\gamma / E_{a} \ln t_{a}$ using linear regression to get $E_{a}$ and its error at low $T_{a}$ [the lowest 5 data points in Fig. 3(c)].

\section{Results for different $K$}

In Fig. 10 we show the collapse of different waiting times $t_{a}$ and the fit of $E_{a}$ at low $T_{a}$ for all ensembles that are not shown in the main text (notably Fig. 3). Note that for $K=3 \times 10^{3}$ we extract $E_{a}$ by directly fitting for low $T_{a}$ for a single $t_{a}$.

\section{Robustness of $E_{a}$}

In Fig. 11 we verify that the consistency with $E_{a} \sim \omega_{c}^{4}$ is robust to a different measure of the softest quasilocalized mode after sample preparation. In particular, we compare with $\omega_{\min }$.
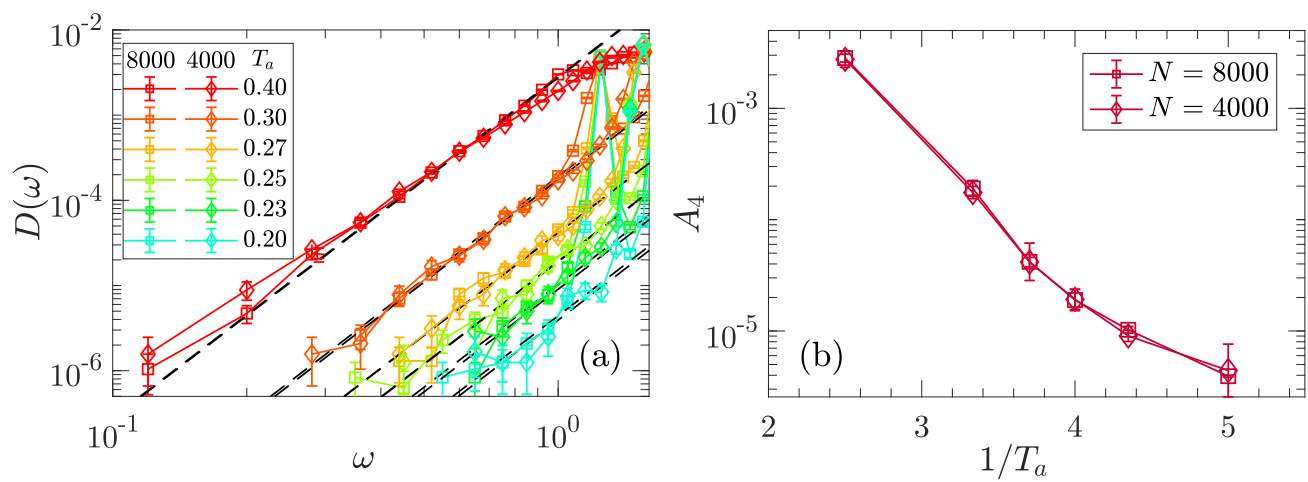

FIG. 9. (a) $D(\omega)$ for different system sizes $N$ and different activation temperatures $T_{a}$ (as indicated in the legend $T_{a}$ increases from bottom to top). (b) $A_{4}$ fitted on (a) as a function of $1 / T_{a}$. Both plots are for $K=10^{2}$ and $t_{a}=500$. 

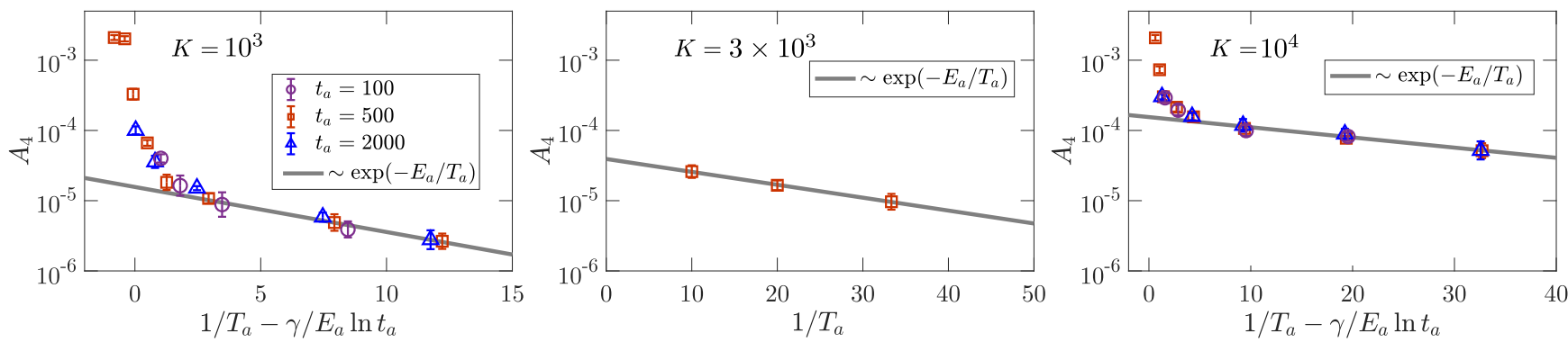

FIG. 10. Fitting of $E_{a}$ and $\gamma$ for all $K$ not shown in the main text. The fitted values are reported in Appendix A.

APPENDIX E: THE JACOBIAN OF THE TRANSFORMATION FROM $\left(\lambda_{1}, \kappa_{1}, \chi_{1}\right)$ to $\left(\lambda_{2}, \kappa_{2}, \chi_{2}\right)$

The potential around the state 1 is given by

$$
E(s)=\frac{1}{2 !} \lambda_{1} s^{2}+\frac{1}{3 !} \kappa_{1} s^{3}+\frac{1}{4 !} \chi_{1} s^{4}+\text { constant }
$$

with the joint distribution $P\left(\lambda_{1}, \kappa_{1}, \chi_{1}\right)$ that is strictly zero at $\lambda_{1}<\omega_{c}^{2}$ and smooth above $\omega_{c}^{2} \cdot \chi_{1}>0$. Without loss of generality, we let $\kappa_{1}>0$. For the new minimum, state 2, the potential reads:

$$
\begin{aligned}
E(s)= & \frac{1}{2 !} \lambda_{2}\left(s+s_{0}\right)^{2}+\frac{1}{3 !} \kappa_{2}\left(s+s_{0}\right)^{3} \\
& +\frac{1}{4 !} \chi_{2}\left(s+s_{0}\right)^{4}+\text { constant }
\end{aligned}
$$

where $s_{0}$ is the shift along $s$, see Fig. 12; and the corresponding joint distribution is $P\left(\lambda_{2}, \kappa_{2}, \chi_{2}\right)$. The convention $\kappa_{1}>0$ leads to the position $s_{2}=-s_{0}$ of state 2 smaller than 0 .

The relation between two sets of coefficients $\lambda_{1}, \kappa_{1}, \chi_{1}$ and $\lambda_{2}, \kappa_{2}, \chi_{2}$ is

$$
\begin{aligned}
& \chi_{1}=\chi_{2} \\
& \kappa_{1}=\kappa_{2}+\chi_{2} s_{0} \\
& \lambda_{1}=\lambda_{2}+\kappa_{2} s_{0}+\frac{1}{2} \chi_{2} s_{0}^{2},
\end{aligned}
$$

where $s_{0}$ as a function of $\left(\lambda_{2}, \kappa_{2}, \chi_{2}\right)$ follows from the fact that the linear term vanishes in Eqs. (E1) and (E2). In particular,

$$
6 \lambda_{2}+3 \kappa_{2} s_{0}+\chi_{2} s_{0}^{2}=0 .
$$

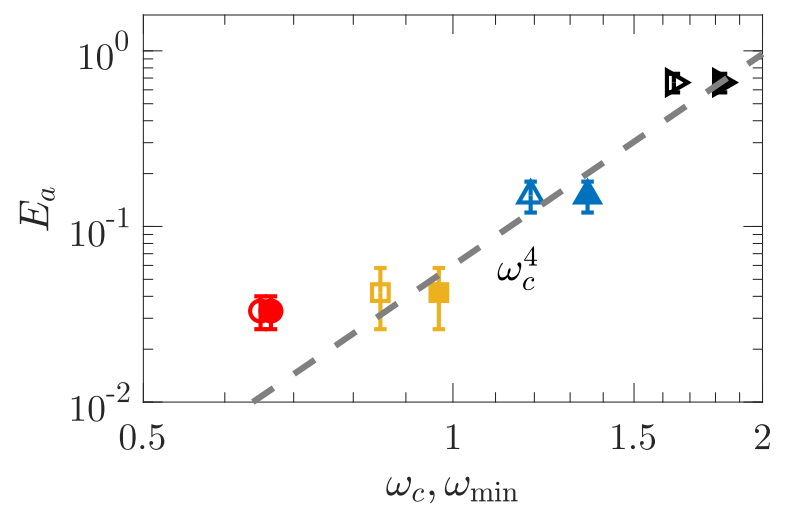

FIG. 11. $E_{a}$ as a function of $\omega_{c}$ (open markers) or as a function of $\omega_{\min }$ (solid markers).
The joint distribution $P\left(\lambda_{2}, \kappa_{2}, \chi_{2}\right)$ is given by

$$
P\left(\lambda_{2}, \kappa_{2}, \chi_{2}\right)=\left|\operatorname{det}\left(\frac{d \lambda_{1} d \kappa_{1} d \chi_{1}}{d \lambda_{2} d \kappa_{2} d \chi_{2}}\right)\right| P\left(\lambda_{1}, \kappa_{1}, \chi_{1}\right),
$$

where

$$
\begin{aligned}
\left|\operatorname{det}\left(\frac{d \lambda_{1} d \kappa_{1} d \chi_{1}}{d \lambda_{2} d \kappa_{2} d \chi_{2}}\right)\right| & \equiv\left|\operatorname{det}\left(\left[\begin{array}{lll}
\frac{\partial \lambda_{1}}{\partial \lambda_{2}} & \frac{\partial \lambda_{1}}{\partial \kappa_{2}} & \frac{\partial \lambda_{1}}{\partial \chi_{2}} \\
\frac{\partial \kappa_{1}}{\partial \lambda_{2}} & \frac{\partial \kappa_{1}}{\partial \kappa_{2}} & \frac{\partial \kappa_{1}}{\partial \chi_{2}} \\
\frac{\partial \chi_{1}}{\partial \lambda_{2}} & \frac{\partial \chi_{1}}{\partial \kappa_{2}} & \frac{\partial \chi_{1}}{\partial \chi_{2}}
\end{array}\right]\right)\right| \\
& =\left|1+\kappa_{2} \frac{\partial s_{0}}{\partial \lambda_{2}}+\chi_{2} \frac{\partial s_{0}}{\partial \kappa_{2}}\right| .
\end{aligned}
$$

From Eq. (E4) we find that

$$
\begin{aligned}
& \frac{\partial s_{0}}{\partial \lambda_{2}}=-\frac{6}{3 \kappa_{2}+2 \chi_{2} s_{0}}, \\
& \frac{\partial s_{0}}{\partial \kappa_{2}}=-\frac{3 s_{0}}{3 \kappa_{2}+2 \chi_{2} s_{0}} .
\end{aligned}
$$

Thus

$$
\left|\operatorname{det}\left(\frac{d \lambda_{1} d \kappa_{1} d \chi_{1}}{d \lambda_{2} d \kappa_{2} d \chi_{2}}\right)\right|=\left|\frac{-3 \kappa_{2}-\chi_{2} s_{0}}{3 \kappa_{2}+2 \chi_{2} s_{0}}\right|=\left|\frac{6 \lambda_{2}}{3 \kappa_{2} s_{0}+2 \chi_{2} s_{0}^{2}}\right| \text {. }
$$

If the excited state of the double-well potential is close to the spinodal case, $\lambda_{2}$ is small (as it is in Fig. 12). In particular, when $\lambda_{2} \approx 0$, it follows that $s_{0} \approx-3 \kappa_{2} / \chi_{2}$. Inserting this in Eq. (E9) gives

$$
\left|\operatorname{det}\left(\frac{d \lambda_{1} d \kappa_{1} d \chi_{1}}{d \lambda_{2} d \kappa_{2} d \chi_{2}}\right)\right| \simeq \frac{2 \lambda_{2} \chi_{2}}{3 \kappa_{2}^{2}} \simeq \frac{\lambda_{2}}{\lambda_{1}} \sim \lambda_{2},
$$

where $\lambda_{1}$ is a large value with the lower bound $\omega_{c}^{2}$. We have thus found that the joint distribution

$$
P\left(\lambda_{2}, \kappa_{2}, \chi_{2}\right) \sim \lambda_{2} P\left(\lambda_{1}, \kappa_{1}, \chi_{1}\right) .
$$

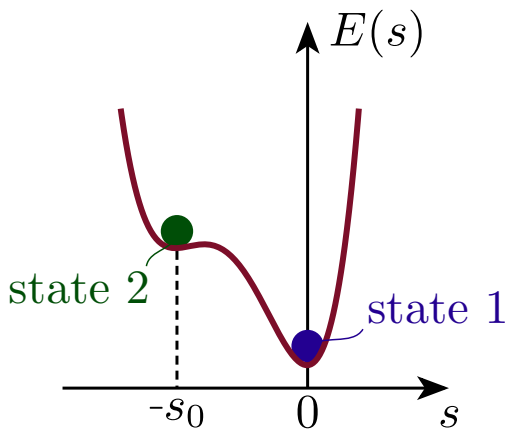

FIG. 12. Double-well potential. 
Hence, the marginal distribution $P\left(\lambda_{2}\right) \sim \lambda_{2}$ (after integrating out $\kappa_{2}$ and $\chi_{2}$ ) and therefore

$$
D\left(\omega_{2}\right)=P\left(\lambda_{2}\right) \frac{d \lambda_{2}}{d \omega_{2}} \sim \omega_{2}^{3} .
$$

\section{Gap in energy barrier distribution}

For a given $\lambda_{1}$ we define $c\left(\kappa_{1}, \chi_{1}\right)=\lambda_{1} \chi_{1} / \kappa_{1}^{2}$, which smoothly varies in a narrow range from $1 / 3$ (for a symmetric double well) to $3 / 8$ (for a spinodal). Then we can express the energy barrier as

$$
\Delta E=\lambda_{1}^{2} \frac{(3-\sqrt{9-24 c})^{2}(-3+12 c+\sqrt{9-24 c})}{192 \chi_{1} c^{2}} .
$$

The function $c$ is slowly varying and not singular so that

$$
\Delta E \sim \lambda_{1}^{2} \sim \omega_{c}^{4} .
$$

Similarly, the energy difference

$$
\begin{aligned}
E_{12} & =\lambda_{1}^{2} \frac{(3+\sqrt{9-24 c})^{2}(-3+12 c-\sqrt{9-24 c})}{192 \chi_{1} c^{2}} \\
& \sim \lambda_{1}^{2} \sim \omega_{c}^{4}
\end{aligned}
$$

(except in the case of a symmetric double well).

\section{APPENDIX F: ESTIMATION OF $\chi_{1}$}

Numerically, the coefficient $\chi_{1}$ [from Eq. (2)] along the direction of the displacement field $\vec{s}$ from the initial minimum to the new minimum whose frequency is smaller than $\omega_{e}$ (the frequency of first tranverse plane waves), can be expressed by the pair interaction $\varphi(\vec{r})$ at mechanical equilibrium, as follows:

$$
\begin{aligned}
\chi_{1}= & \sum_{\alpha, \beta, \eta, v=1}^{3} \sum_{n, m, k, l=1}^{N} \frac{\partial^{4} U}{\partial r_{n}^{\nu} \partial r_{m}^{\eta} \partial r_{k}^{\beta} \partial r_{l}^{\alpha}} s_{l}^{\alpha} s_{k}^{\beta} s_{m}^{\eta} s_{n}^{\nu} \\
= & \sum_{i<j}\left\{\left(\frac{1}{r_{i j}^{4}} \frac{d^{4} \varphi}{d r^{4}}-\frac{6}{r_{i j}^{5}} \frac{d^{3} \varphi}{d r^{3}}+\frac{15}{r_{i j}^{6}} \frac{d^{2} \varphi}{d r^{2}}-\frac{15}{r_{i j}^{7}} \varphi^{\prime}\right)\left(\vec{r}_{i j} \cdot \vec{s}_{i j}\right)^{4}\right. \\
& +\left(\frac{1}{r_{i j}^{3}} \frac{d^{3} \varphi}{d r^{3}}-\frac{3}{r_{i j}^{4}} \frac{d^{2} \varphi}{d r^{2}}+\frac{3}{r_{i j}^{5}} \varphi^{\prime}\right) 6\left(\vec{r}_{i j} \cdot \vec{s}_{i j}\right)^{2}\left(\vec{s}_{i j} \cdot \vec{s}_{i j}\right) \\
& \left.+\left(\frac{1}{r_{i j}^{2}} \frac{d^{2} \varphi}{d r^{2}}-\frac{1}{r_{i j}^{3}} \varphi^{\prime}\right) 3\left(\vec{s}_{i j} \cdot \vec{s}_{i j}\right)^{2}\right\},
\end{aligned}
$$

where $\vec{r}_{i}$ are the particles' equilibrium positions, $\vec{s}$ is the direction (the normalized displacement field from the quenched states before and after reheating), and $\vec{s}_{i j}=\vec{s}_{i}-\vec{s}_{j}$.

From the results in Fig. 13 we observe that at small $\lambda_{1}, \chi_{1}$ is independent of $\lambda_{1}$. Hence, we regard it as a constant. Here we estimate $\chi_{1} \approx 1500$ by its median value. Note that $\chi_{1}$ has unit $m \omega_{0}^{2} / d_{0}^{2}$, where $m$ is the particle mass, $d_{0}$ is approximately equal to the interparticle distance $a$ (hence we take $a=d_{0}$ ), and $\omega_{0} \equiv 1 / t_{0}$ is the unit frequency in our simulation which is about $\omega_{D} / 18$. Here $\omega_{D}$ is the Debye frequency. Hence we get $\chi_{1} \approx 1500 m \omega_{0}^{2} / a^{2} \approx 4.6 m \omega_{D}^{2} / a^{2}$.

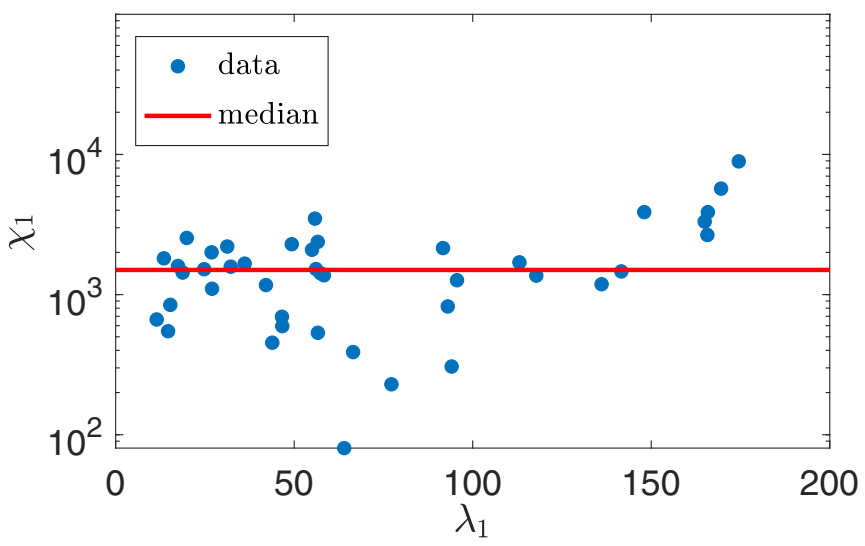

FIG. 13. Scatter plot $\chi_{1}$ as function of $\lambda_{1}$ for our largest gap $\left(\omega_{c}=1.64\right) . \lambda_{1}$ follows as $\lambda_{1}=\sum_{\alpha, \beta=1}^{3} \sum_{k, l=1}^{N} s_{l}^{\alpha} H_{l k}^{\alpha \beta} s_{k}^{\beta}$.

The relation between $\omega_{D}$ and $\omega_{0}$ is calculated by $\omega_{D}=$ $\left(9 N /\left(4 \pi\left(2 \omega_{e}^{-3}+\omega_{l}^{-3}\right)\right)\right)^{1 / 3} \approx 18 \omega_{0}$, where $\omega_{e}=1.26 \omega_{0}$ and $\omega_{l} \approx 2.5 \omega_{e}$ ( $\omega_{l}$ is the frequency of first longitudinal modes). Note that both are plane waves. In particular, the first transverse modes consist of 12 modes and the first longitudinal modes consists of six modes. Their identification is straightforward through the number of modes and their participation ratios, which are around 0.6. Note that $\omega_{0}=1$ in our simulation, so $\omega_{D} \approx 18$ and $\omega_{c} / \omega_{D} \approx 0.1$.

\section{APPENDIX G: GEOMETRY OF REARRANGEMENTS}

\section{Protocol to separate rearrangements}

The displacement field between the states before and after reheating may contain more than one elementary excitation. We extract them one-by-one from this displacement field, by assuming them linearly independent. This corresponds to the following algorithm:

(1) Find the particle with the largest displacement.

(2) Place a small sphere centered at this particle with a radius $\tilde{R}^{(i)}=(V / N)^{1 / 3}$ (with $i$ the increment number, starting at $i=0$ ).

(3) Set all displacements outside the sphere equal to zero. The particle displacements inside the sphere are not changed.

(4) Minimize the energy $U$ (every particle is free to move).

(5) Increase the radius of sphere: $\tilde{R}^{(i+1)}=\tilde{R}^{(i)}+\Delta \tilde{R}$, and reset the displacements as in step 3 (the particle displacements outside the sphere are set to zero and those inside the sphere equal to the original particle displacements).

(6) Repeat steps 4 and 5, until the localized mode is identified. In particular, stop when $\left|U^{(i+1)}-U^{(i)}\right|<10^{-6}$ and, to avoid stopping too early, the norm of the displacement field is larger than $10^{-2}$. Note that $U^{(i)}$ refers to the potential energy after energy minimization, in step 4 , for increment $i$.

The local rearrangement is then the displacement field after the last energy minimization. We then subtract it from the original displacement and continue to extract the next elementary excitation, by repeating this algorithm. We continue to do so until we have extracted all elementary excitations. In particular, we stop $\tilde{R}^{(i)} / L>\sqrt{3} / 2$ (with $L$ the linear size of the simulation box). 

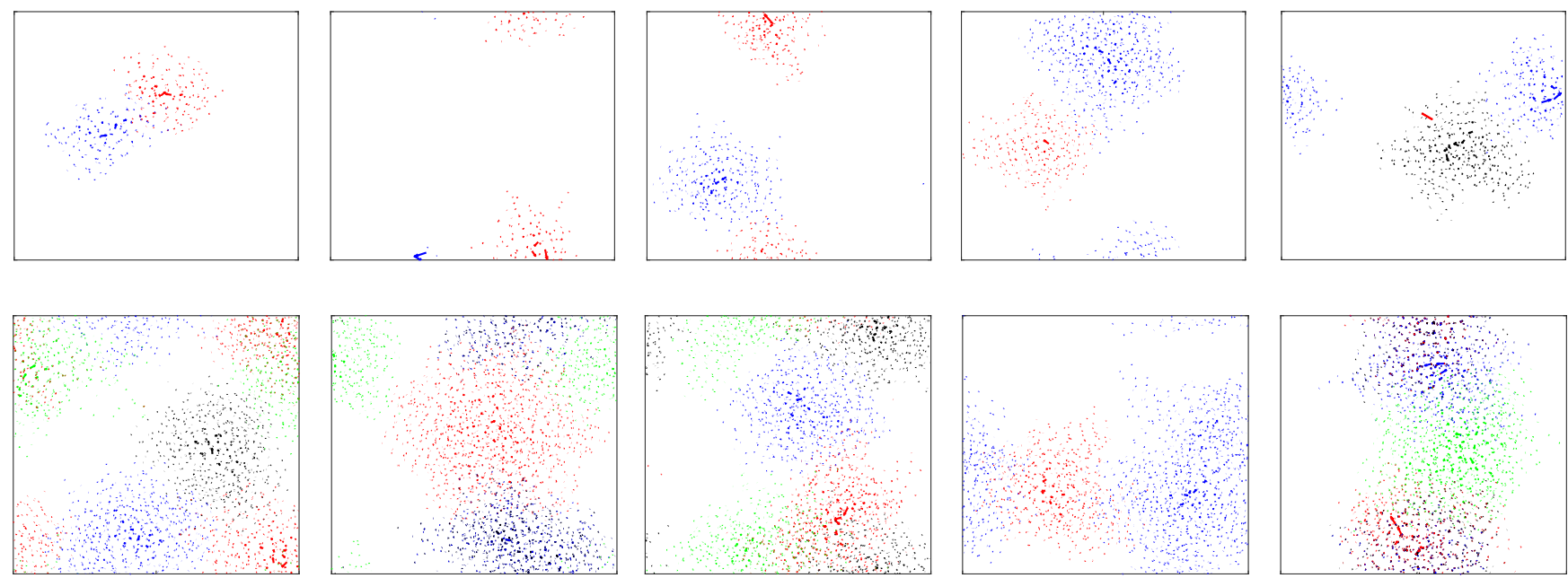

FIG. 14. Individual local rearrangements projected on the $x y$ plane, shown using different colors, in five randomly chosen examples (from those samples that show more than one local rearrangement) for: (top) $K=10^{2}, T_{a}=0.15$, and $t_{a}=500$, (bottom) $K=10^{4}, T_{a}=0.03$, and $t_{a}=500$.

\section{Results}

Five representative samples (for two different $K$ ) showing our separation protocol are shown in Fig. 14, whereby the displacement field of each elementary excitation is plotted using a different color. On average, we measure $\{1.4,1.6,1.9,3.5\}$ elementary excitations for $K=\left\{10^{2}, 10^{3}, 3 \times 10^{3}, 10^{4}\right\}$ at the lowest thermal activation $T_{a}=\{0.15,0.07,0.03,0.03\}$ with $t_{a}=500$.

We, furthermore, include the distribution of the participation ratio of the elementary excitations in Fig. 15(a), whereby the different colors correspond to the different data points in Fig. 5(a). We observe that the elementary excitations become more localized for larger gaps. Likewise, we include the distribution of the maximum displacement of each elementary excitation in Fig. 15(b) [the different colors correspond to the different data points in Fig. 5(b)]. In this case we observe that the maximum displacement increases for our largest $\omega_{c}$ (in black) as the result of stringlike motion. This is supported by the distinct part of the Van Hove correlation in Fig. 15(c) that displays a sharp peak around $r=0$ only for our largest $\omega_{c}$ (in black). For this configuration, we plot the distribution of the number of permuting particles, $\# n_{p}$, inside the string in Fig. 15(d).
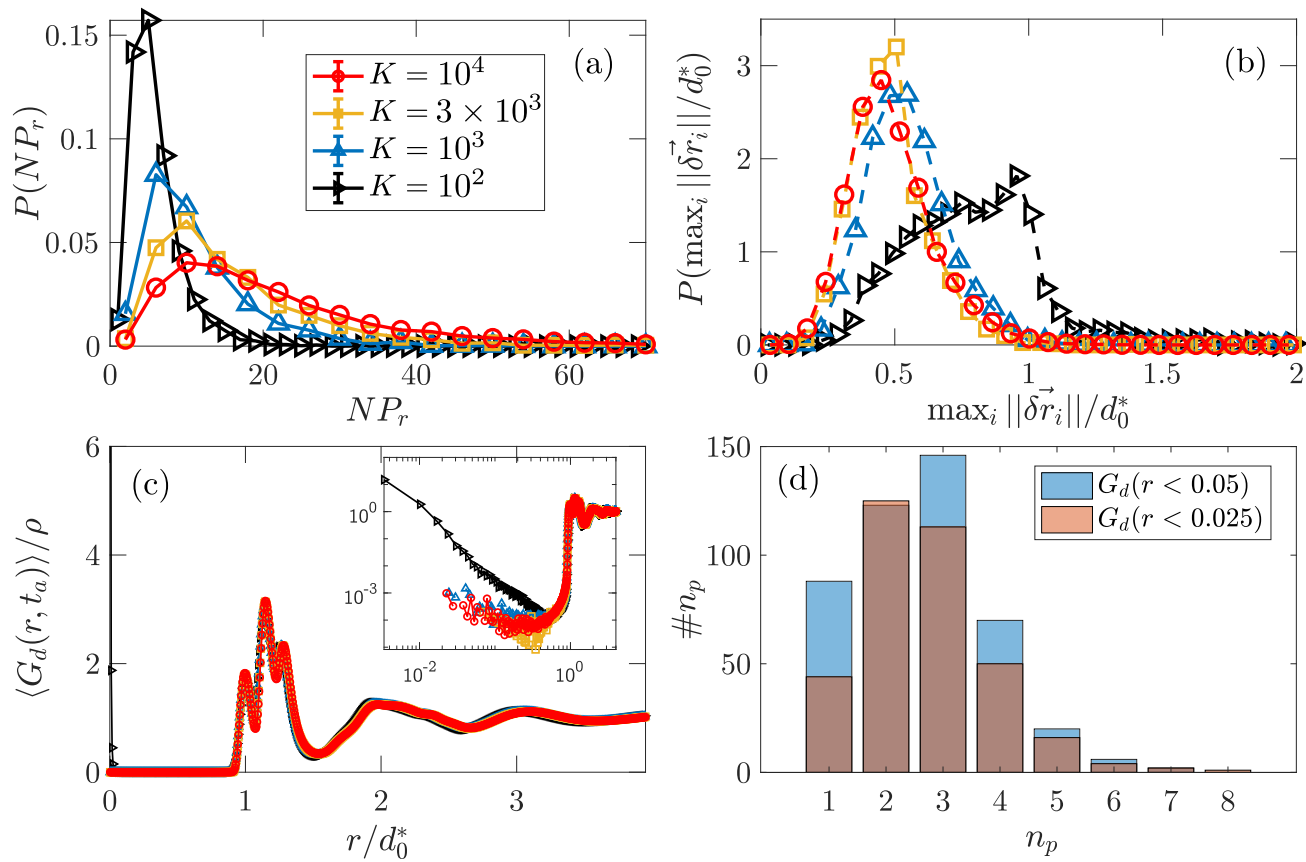

FIG. 15. Probability distribution of (a) the participation ratio $N P_{r}$ and (b) the maximal particle displacement $\max _{i}\left\{\left\|\delta \vec{r}_{i}\right\|\right\}$ at different $\omega_{c}$. (c) The distinct part of the Van Hove correlation normalised by the number density $\left\langle G_{d}\right\rangle / \rho$ at different $\omega_{c}$. (d) Histogram of the number of particles that permute per realisation, $\# n_{p}$ (for largest $\omega_{c}$ ) at two different cutoff distances $r_{c} / d_{0}^{*}=\{0.025,0.05\}$. 
[1] W. Phillips, J. Low Temp. Phys. 7, 351 (1972).

[2] P. Anderson, B. Halperin, and C. Varma, Philos. Mag. 25, 1 (1972).

[3] W. Phillips, Rep. Prog. Phys. 50, 1657 (1987).

[4] C. Donati, J. F. Douglas, W. Kob, S. J. Plimpton, P. H. Poole, and S. C. Glotzer, Phys. Rev. Lett. 80, 2338 (1998).

[5] H.-B. Yu, R. Richert, and K. Samwer, Sci. Adv. 3, e1701577 (2017).

[6] C. Maloney and A. Lemaître, Phys. Rev. Lett. 93, 016001 (2004).

[7] A. Heuer, J. Phys. Condens. Matter 20, 373101 (2008).

[8] A. Argon, Acta Metall. 27, 47 (1979).

[9] D. Vandembroucq and S. Roux, Phys. Rev. B 84, 134210 (2011).

[10] M. Ozawa, L. Berthier, G. Biroli, A. Rosso, and G. Tarjus, Proc. Natl. Acad. Sci. 115, 6656 (2018).

[11] M. Popović, T. W. J. de Geus, and M. Wyart, Phys. Rev. E 98, 040901(R) (2018).

[12] M. Ediger, C. Angell, and S. Nagel, J. Phys. Chem. 100, 13200 (1996).

[13] D. R. Queen, X. Liu, J. Karel, T. H. Metcalf, and F. Hellman, Phys. Rev. Lett. 110, 135901 (2013).

[14] T. Pérez-Castañeda, C. Rodríguez-Tinoco, J. Rodríguez-Viejo, and M. Ramos, Proc. Natl. Acad. Sci. 111, 11275 (2014).

[15] J. M. Martinis, K. B. Cooper, R. McDermott, M. Steffen, M. Ansmann, K. D. Osborn, K. Cicak, S. Oh, D. P. Pappas, R. W. Simmonds, and C. C. Yu, Phys. Rev. Lett. 95, 210503 (2005).

[16] D. A. Parshin, H. R. Schober, and V. L. Gurevich, Phys. Rev. B 76, 064206 (2007).

[17] W. Ji, M. Popović, T. W. J. de Geus, E. Lerner, and M. Wyart, Phys. Rev. E 99, 023003 (2019).

[18] H. Schober, C. Oligschleger, and B. Laird, J. Non. Cryst. Solids 156-158, 965 (1993).

[19] M. Baity-Jesi, V. Martín-Mayor, G. Parisi, and S. Perez-Gaviro, Phys. Rev. Lett. 115, 267205 (2015).

[20] E. Lerner, G. Düring, and E. Bouchbinder, Phys. Rev. Lett. 117, 035501 (2016).

[21] H. Mizuno, H. Shiba, and A. Ikeda, Proc. Natl. Acad. Sci. 114, E9767 (2017).

[22] E. Lerner and E. Bouchbinder, J. Chem. Phys. 148, 214502 (2018).

[23] M. Shimada, H. Mizuno, M. Wyart, and A. Ikeda, Phys. Rev. E 98, 060901(R) (2018).

[24] C. Scalliet, L. Berthier, and F. Zamponi, Nat. Commun. 10, 5102 (2019).

[25] L. Wang, A. Ninarello, P. Guan, L. Berthier, G. Szamel, and E. Flenner, Nat. Commun. 10, 26 (2019).

[26] Obtained by rapidly quenching an equilibrated liquid (at initial temperature $T$ ) to zero temperature.

[27] C. Rainone, E. Bouchbinder, and E. Lerner, Proc. Natl. Acad. Sci. 117, 5228 (2020).

[28] D. Khomenko, C. Scalliet, L. Berthier, D. R. Reichman, and F. Zamponi, Phys. Rev. Lett. 124, 225901 (2020).

[29] V. L. Gurevich, D. A. Parshin, and H. R. Schober, Phys. Rev. B 67, 094203 (2003).

[30] V. Gurarie and J. T. Chalker, Phys. Rev. B 68, 134207 (2003).

[31] A. Cavagna, Phys. Rep. 476, 51 (2009).

[32] V. Lubchenko and P. Wolynes, Annu. Rev. Phys. Chem. 58, 235 (2007).
[33] G. Biroli and J.-P. Bouchaud, in Structural Glasses and Supercooled Liquids: Theory, Experiment, and Applications, edited by P. G. Wolynes and V. Lubchenko (John Wiley \& Sons, Inc., Hoboken, New Jersey, 2012).

[34] G. Parisi, J. Phys. Condens. Matter 15, S765 (2003).

[35] G. Kapteijns, W. Ji, C. Brito, M. Wyart, and E. Lerner, Phys. Rev. E 99, 012106 (2019).

[36] Breathing particles is an alternative version of swap algorithms in which particles of different radii are exchanged [59].

[37] C. Brito, E. Lerner, and M. Wyart, Phys. Rev. X 8, 031050 (2018).

[38] E. Lerner and E. Bouchbinder, Phys. Rev. E 96, 020104(R) (2017).

[39] L. Berthier, E. Flenner, C. Fullerton, C. Scalliet, and M. Singh, J. Stat. Mech. Theory Exp. (2019) 064004.

[40] E. Bitzek, P. Koskinen, F. Gähler, M. Moseler, and P. Gumbsch, Phys. Rev. Lett. 97, 170201 (2006).

[41] We define a rearrangement by a finite norm of the displacement field that results from reheating, see Appendix A for details.

[42] For our system size, at $t_{a}=500$ (the time scale we use later), the temperature for which we have in average one rearrangement per realization $T_{a}^{*}\left(\omega_{c}=1.64\right) \approx 0.17$, which we estimate using the fit of the Arrhenius-like behavior below.

[43] Such interactions are relevant even at high temperature near the glass transition [63]. Note that when quenching the system to zero temperature after a reheating, interactions with relaxing vibrational modes may also destabilise excitations which are close to their spinodal.

[44] Interactions between excitations cause a pseudogap in the density $P(x) \sim x^{\theta}$ of excitations within a force $x$ to fail $[64,65]$. Near a saddle-node bifurcation, one has $\omega \sim x^{1 / 4}$ leading to $D_{L}(\omega) \sim \omega^{\alpha}$ where $\alpha=3+4 \theta$. Empirically $\theta \approx 0.3$ after a slow quench from high temperature (but it is larger for a fast quench) [17], which would lead to an exponent $\alpha \approx 4.2$ consistent with our measurement.

[45] We observed that for small gaps the assumption that all the excitations are in their energy minimum breaks down, but on the contrary for our largest gap it holds true for about $90 \%$ of excitations causing quasilocalised modes. This state of affairs is expected since for very small gaps particles can hardly breathe, and the corresponding gapped states obtained by our protocol are not extremely stable.

[46] Shear stress is measured relative to the initial value at $\epsilon=0$.

[47] D. Parshin, Phys. Scr. T49A, 180 (1993).

[48] M. Mertig, G. Pompe, and E. Hegenbarth, Solid State Commun. 49, 369 (1984).

[49] L. Yan, E. DeGiuli, and M. Wyart, Europhys. Lett. 114, 26003 (2016).

[50] E. Lerner, E. DeGiuli, G. Düring, and M. Wyart, Soft Matter 10, 5085 (2014).

[51] In general to create gapped glasses one needs to use a gradient descent method using swap or equivalently breathing particles up to zero temperature. If instead one uses MD with the normal dynamics to quench from a finite temperature, the gap of magnitude $\omega_{c}$ will be filled by excitations .

[52] M. Goldstein, J. Chem. Phys. 51, 3728 (1969).

[53] T. Hecksher and J. Dyre, J. Non. Cryst. Solids 407, 14 (2015).

[54] Y. Jin, P. Urbani, F. Zamponi, and H. Yoshino, Sci. Adv. 4, eaat6387 (2018). 
[55] See Ref. [27] for a discussion on quasilocalized modes. Concerning TLS, a smaller participation ratio suggests a higher tunneling amplitude, which may in turn affect the TLS density.

[56] It was argued within RFOT that strings would exist at intermediate temperatures, [66,67]. Yet, this analysis is based on the "library of states" picture that describes only rough barriers consisting of many intermediary ones (that must exist between each state of the library), leading to impact rearrangements. This description thus cannot apply to the elementary excitations displaying a single barrier studied here. This picture is explicitly different than our view. In our view, a description of strings should also explain why particles exchange positions.

[57] M. Wyart and M. E. Cates, Phys. Rev. Lett. 119, 195501 (2017).

[58] H. Ikeda, F. Zamponi, and A. Ikeda, J. Chem. Phys. 147, 234506 (2017).

[59] A. Ninarello, L. Berthier, and D. Coslovich, Phys. Rev. X 7, 021039 (2017).
[60] I. Lifshitz, Sov. Phys. Usp. 7, 549 (1965).

[61] S. Alexander, Phys. Rep. 296, 65 (1998).

[62] M. Wyart, L. E. Silbert, S. R. Nagel, and T. A. Witten, Phys. Rev. E 72, 051306 (2005).

[63] A. Lemaître, Phys. Rev. Lett. 113, 245702 (2014).

[64] J. Lin, A. Saade, E. Lerner, A. Rosso, and M. Wyart, Europhys. Lett. 105, 26003 (2014).

[65] J. Lin and M. Wyart, Phys. Rev. X 6, 011005 (2016).

[66] J. Stevenson, J. Schmalian, and P. Wolynes, Nat. Phys. 2, 268 (2006).

[67] J. Stevenson and P. Wolynes, Nat. Phys. 6, 62 (2010).

[68] H. Berendsen, J. Postma, W. van Gunsteren, A. DiNola, and J. Haak, J. Chem. Phys. 81, 3684 (1984).

[69] For $K=10^{3}, \omega_{e}=1.30 \pm 0.02$, and for $K=10^{2}, \omega_{e}=$ $\{1.26 \pm 0.03,1.73 \pm 0.05\}$ (where the uncertainty refers to the standard deviation). 